\title{
IL-6R/STAT3/miR-34a feedback loop promotes EMT-mediated colorectal cancer invasion and metastasis
}

\author{
Matjaz Rokavec, ${ }^{1}$ Meryem Gülfem Öner, ${ }^{1,2}$ Huihui Li, ${ }^{1}$ Rene Jackstadt,, ${ }^{1}$ Longchang Jiang, ${ }^{1}$ \\ Dmitri Lodygin, ${ }^{3}$ Markus Kaller, ${ }^{1}$ David Horst, ${ }^{4}$ Paul K. Ziegler, ${ }^{2}$ Sarah Schwitalla, ${ }^{2}$ \\ Julia Slotta-Huspenina, ${ }^{5}$ Franz G. Bader, ${ }^{6}$ Florian R. Greten, ${ }^{2,7,8,9}$ and Heiko Hermeking, ${ }^{1,8}$
}

\begin{abstract}
${ }^{1}$ Experimental and Molecular Pathology, Institute of Pathology, Ludwig-Maximilians-University Munich, Munich, Germany. ${ }^{2}$ Institute of Molecular Immunology, Klinikum Rechts der Isar, Technical University Munich, Munich, Germany. ${ }^{3}$ Institute for Multiple Sclerosis Research and Department of Neuroimmunology, Gemeinnützige Hertie-Stiftung, and University Medical Center Göttingen, Göttingen, Germany. ${ }^{4}$ Institute of Pathology, Ludwig-Maximilians-University Munich, Munich, Germany. Institute of Pathology and ${ }^{5}$ Department of General Surgery, Klinikum Rechts der Isar, Technical University Munich, Munich, Germany. ${ }^{7}$ Georg-Speyer-Haus, Institute for Tumor Biology and Experimental Therapy, Frankfurt, Germany. ${ }^{8}$ German Cancer Consortium (DKTK), Heidelberg, Germany. ${ }^{9}$ German Cancer Research Center (DKFZ), Heidelberg, Germany.
\end{abstract}

\begin{abstract}
Members of the miR-34 family are induced by the tumor suppressor p53 and are known to inhibit epithelialto-mesenchymal transition (EMT) and therefore presumably suppress the early phases of metastasis. Here, we determined that exposure of human colorectal cancer (CRC) cells to the cytokine IL-6 activates the oncogenic STAT3 transcription factor, which directly represses the MIR34A gene via a conserved STAT3-binding site in the first intron. Repression of MIR34A was required for IL-6-induced EMT and invasion. Furthermore, we identified the IL-6 receptor (IL-6R), which mediates IL-6-dependent STAT3 activation, as a conserved, direct miR-34a target. The resulting IL-6R/STAT3/miR-34a feedback loop was present in primary colorectal tumors as well as CRC, breast, and prostate cancer cell lines and associated with a mesenchymal phenotype. An active IL-6R/ STAT3/miR-34a loop was necessary for EMT, invasion, and metastasis of CRC cell lines and was associated with nodal and distant metastasis in CRC patient samples. p53 activation in CRC cells interfered with IL-6-induced invasion and migration via miR-34a-dependent downregulation of IL6R expression. In Mir34a-deficient mice, colitis-associated intestinal tumors displayed upregulation of p-STAT3, IL-6R, and SNAIL and progressed to invasive carcinomas, which was not observed in WT animals. Collectively, our data indicate that p53-dependent expression of miR-34a suppresses tumor progression by inhibiting a IL-6R/STAT3/miR-34a feedback loop.
\end{abstract}

\section{Introduction}

Cancer is one of the major health problems in the Western world, with metastasis being the most critical concern, because it accounts for over $90 \%$ of cancer mortality (1). Primary colorectal cancer (CRC) originates from epithelial cells that line the gastrointestinal tract (2). During progression to metastasis, cancer cells are thought to acquire a mesenchymal phenotype, which allows them to leave the site of the primary tumor, invade surrounding tissues, and migrate to distant organs. After seeding, these cells switch back to an epithelial phenotype and proliferate to form metastases (3). The processes by which cells switch between epithelial and mesenchymal phenotypes are known as the epithelial-to-mesenchymal transition (EMT) and its counterpart, the mesenchymalto-epithelial transition (MET) (4).

Growing evidence suggests that inflammation promotes EMT (5). Inflammation is a biological process that is initiated upon injury to remove harmful stimuli and to initiate a healing process (6). However, if inflammation is prolonged, it may be harmful to the organism and promote permanent disease states, including cancer. About $25 \%$ of cancers appear due to chronic infection or other types of chronic inflammation (7). For example, inflammatory bowel disease or colitis are important risk factors for

Authorship note: Matjaz Rokavec, Meryem Gülfem Öner, Huihui Li, and Rene Jackstadt contributed equally to this work.

Conflict of interest: The authors have declared that no conflict of interest exists. Citation for this article: J Clin Invest. 2014;124(4):1853-1867. doi:10.1172/JCI73531.
CRC (8). However, by now, it is accepted that basically all solid tumors - even those arising in the absence of chronic inflammation - contain an inflammatory microenvironment (9). Recently, inflammation caused by a barrier defect, which is characteristic for the majority of sporadic CRCs, was shown to play a major role in the initiation and progression of CRC $(10,11)$. An important mediator of the tumor-promoting effects of inflammation is IL-6, a proinflammatory cytokine that is produced by several types of immune cells and carcinomas, especially in later states of tumorigenesis (12). Elevated levels of IL-6 in serum have been associated with advanced stages and increased size of tumors, metastasis, and decreased survival of CRC patients (13), yet the molecular mechanisms underlying these associations are not well understood.

Numerous studies have documented that miRNAs have important regulatory functions in biological processes that represent the hallmarks of cancer, such as proliferation, apoptosis, invasion, and metastasis (14). Moreover, miRNAs belonging to the miR-200 and miR-34 families inhibit metastasis, presumably by reversing EMT and promoting MET $(15,16)$. The miR-34 family members display the most significant induction by p53 among all miRNAs (17), and miR-34a and miR-34b/c are involved in the suppression of EMT by directly inhibiting the expression of the EMT-inducing transcription factor (EMT-TF) SNAIL $(18,19)$. miRNAs frequently form feedback loops, since they are themselves regulated by transcription factors, which they directly or indirectly target $(20,21)$. Such self-stabilizing circuits can be central components of epigenetic switches, where cellular phenotypes and expression patterns 
convert from one stable epigenetic state to another without changes in DNA sequence (22). Epigenetic switches require an initiating event (i.e., inflammation), but the phenotypes of the new cell type are inherited in the absence of the initiating signal and maintained by self-sustained feedback loops (23). Recently, it has been shown that inflammation-induced epigenetic switches promote cancer initiation $(24,25)$.

In this study, we investigated whether inflammation-induced epigenetic switches also contribute to cancer progression. We uncovered a feedback circuit that is established by IL-6/STAT3-mediated repression of MIR34A and upregulation of IL-6R, which promotes and maintains EMT, invasiveness, and metastasis in CRC.

\section{Results}

IL-6 induces EMT, invasiveness, and metastatic properties of CRC cells. To determine whether IL-6 induces EMT in CRC cells, we treated the human CRC cell line DLD-1, which exhibits an epithelial phenotype (see also ref. 26), with recombinant IL-6. As expected, STAT3, an effector of IL-6 signaling, was phosphorylated and therefore presumably activated after treatment of DLD- 1 cells with IL-6 (Supplemental Figure 1A; supplemental material and uncut Western blot membranes are available online with this article; doi:10.1172/JCI73531DS1) in a bimodal kinetic, which is typical for IL-6-mediated STAT3 activation (27). Treatment of DLD-1 cells with IL-6 resulted in EMT, as evidenced by induction of the mesenchymal markers Vimentin (VIM), SNAIL, SLUG, and ZEB1 and repression of the epithelial marker E-cadherin (CDH1) (Figure 1A and Supplemental Figure 1, B and C). IL-6 treatment also caused a rearrangement of F-actin stress fibers in the cytoplasm, another feature of EMT (28) (Supplemental Figure 1C). During cancer progression, EMT may contribute to increased invasiveness of cancer cells (29). Indeed, treatment of DLD-1 cells with IL-6 resulted in increased invasiveness, which was prevented by RNA interference-mediated downregulation of STAT3 or IL- 6 receptor (IL-6R) expression (Figure 1B and Supplemental Figure 1D). Ex vivo treatment with IL- 6 also promoted lung metastasis formation of DLD-1 cells injected into the tail veins of NOD/SCID mice, as determined by longitudinal in vivo imaging of DLD-1 cells stably expressing a luciferase marker gene over a period of 10 weeks (Figure 1C). After 10 weeks, mice were sacrificed and lung metastases were confirmed using H\&E staining (Supplemental Figure $1 \mathrm{E})$. Moreover, lungs of mice injected with IL-6-treated DLD-1 cells showed an increased number of metastatic tumor nodules (Supplemental Figure 1F). In summary, these results show that DLD- 1 cells undergo EMT in response to IL-6 exposure, which is accompanied by an increase in metastatic capacity.

IL-6-induced EMT and invasion of CRC cells are mediated by direct repression of $m i R-34$ a by STAT3. We have previously shown that repression of the microRNA MIR34A by the EMT-TF SNAIL critically contributes to EMT and associated traits in CRC cells (18). In order to determine whether IL-6-induced EMT involves repression of MIR34A as well, we analyzed miR-34a expression in DLD-1 cells after IL- 6 treatment. Indeed, the expression of primary and mature miR-34a decreased after exposure to IL- 6 in a time-dependent manner (Figure 1D). miR-34a was also repressed after exposure of HT-29 CRC and MCF7 breast cancer cells to IL-6 (Figure 1E). Therefore, this effect is not restricted to DLD-1 cells, but is presumably a general response of epithelial cells. The levels of miR-34b and miR-34c expression decreased after IL-6 treatment as well, although not in a statistically significant man- ner (Supplemental Figure 2A). Inspection of the MIR34A genomic region revealed a phylogenetically conserved STAT3-binding site located in the first intron in close proximity to the first exon (Figure $1 \mathrm{~F}$ ), whereas the $M I R 34 B / C$ promoter region was devoid of STAT3-binding motifs (data not shown). After treatment of DLD-1 cells with IL- 6 for 20 minutes, the STAT3 occupancy at the MIR34A promoter significantly increased, as shown by ChIP (Figure 1G). Moreover, siRNA-mediated downregulation of STAT3 prevented the repression of MIR34A after IL- 6 treatment, demonstrating that STAT3 mediates the repression of MIR34A observed after IL-6 exposure (Figure 1H). Ectopic expression of miR-34a from an episomal, DOX-inducible vector prevented IL-6-induced EMT of DLD-1 cells as indicated by the absence of characteristic differential expression of the EMT markers CDH1, VIM, SNAIL, SLUG, and ZEB1 (Figure 1I and Supplemental Figure 2B). Furthermore, ectopic miR-34a also blocked IL-6induced invasion of DLD-1 cells (Figure 1J). Interestingly, in cells explanted from lung metastases that formed from IL-6-treated DLD-1 cells, the expression of miR-34a was similar to that in the parental DLD-1 cells and therefore higher than in IL-6-treated cells at the time point of tail-vein injection (Supplemental Figure 2C). Likewise, the expression of EMT markers in cells explanted from metastases was comparable to the parental DLD-1 cells (Supplemental Figure 2C), suggesting that cells had undergone MET during the outgrowth of lung metastases in mice. In summary, downregulation of MIR34A by STAT3 is required for IL-6induced EMT and invasion.

We have previously shown that SNAIL and miR-34a repress each other in a double-negative feedback loop (18). To investigate a potential synergistic cooperation between the STAT3 and SNAIL with respect to the regulation of miR-34a expression, we downregulated SNAIL expression with siRNAs in DLD-1 cells and subsequently treated them with IL-6 (Supplemental Figure $2 \mathrm{D})$. Suppression of SNAIL only resulted in a slight, nonsignificant reduction of IL-6-mediated miR-34a repression (Supplemental Figure 2, E and H), suggesting that direct repression of miR-34a by STAT3 prevails and cannot be overcome by silencing of SNAIL. However, SNAIL repression counteracted the IL-6mediated repression of CDH1 (Supplemental Figure 2, F and $\mathrm{H})$ and markedly reduced the IL-6-mediated induction of VIM (Supplemental Figure 2, G and H), suggesting that SNAIL is an important downstream effector of IL-6/STAT3/miR-34a signaling with respect to EMT.

IL6R is a direct target of miR-34. Since miRNAs are often components of feedback loops, we hypothesized that miR-34a itself may target components of the JAK/STAT pathway. Indeed, by using the miRNA target prediction software Target Scan, we identified the STAT3, JAK2, and IL6R mRNAs as putative miR-34a targets (data not shown). To validate them as miR-34a targets, we utilized SW480 CRC cells, which express low levels of endogenous miR-34a, and stably transfected them with an episomal plasmid allowing DOX-inducible miR-34a expression. Ectopic expression of miR-34a in SW480 cells for 48 hours resulted in the repression of IL6R, but not JAK2 or STAT3 mRNA (Figure 2A). Moreover, ectopic miR-34a repressed IL6R mRNA and protein in a time-dependent manner (Figure 2, B and C). Besides the membrane-bound IL-6R ( $\mathrm{m}$-IL-6R) we also observed a signal at $50 \mathrm{kDa}$, which corresponds to the soluble IL-6R (s-IL-6R) (30). Densitometric quantification of IL-6R signals with normalization to $\beta$-actin showed a statistically significant decrease of both m-IL-6R and s-IL-6R 
A

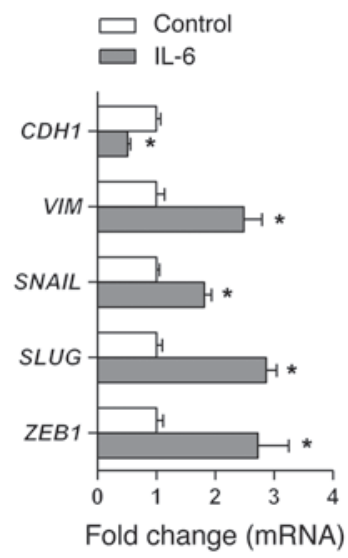

D

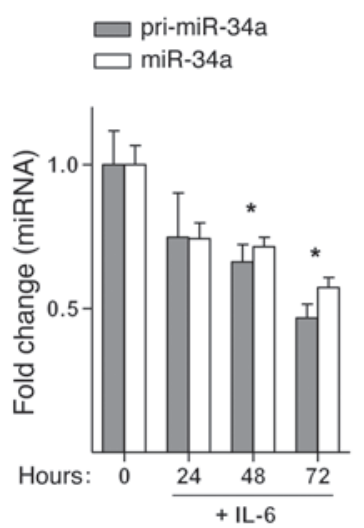

H

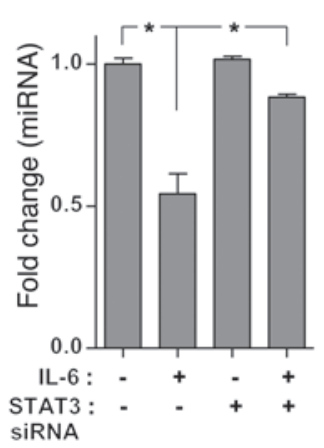

B

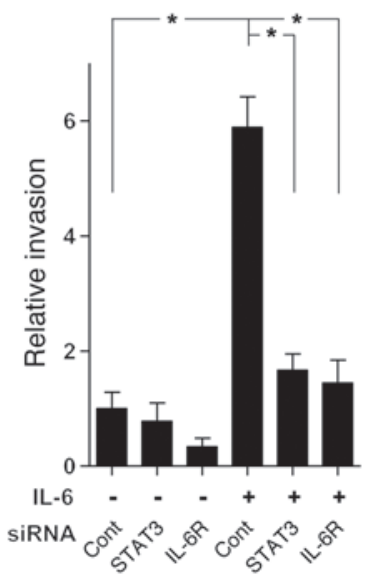

$\mathbf{E}$

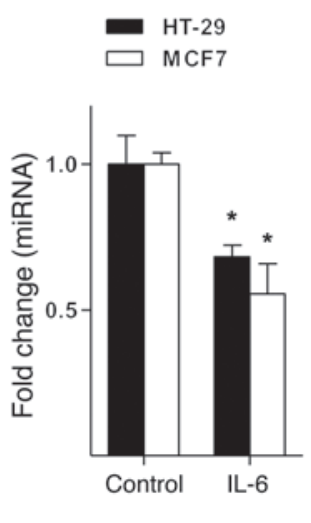

I DLD-1/pRTR-miR-34a

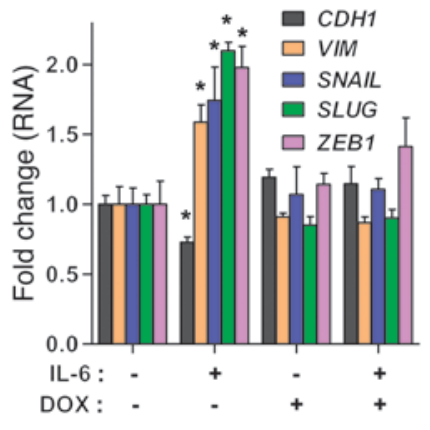

C
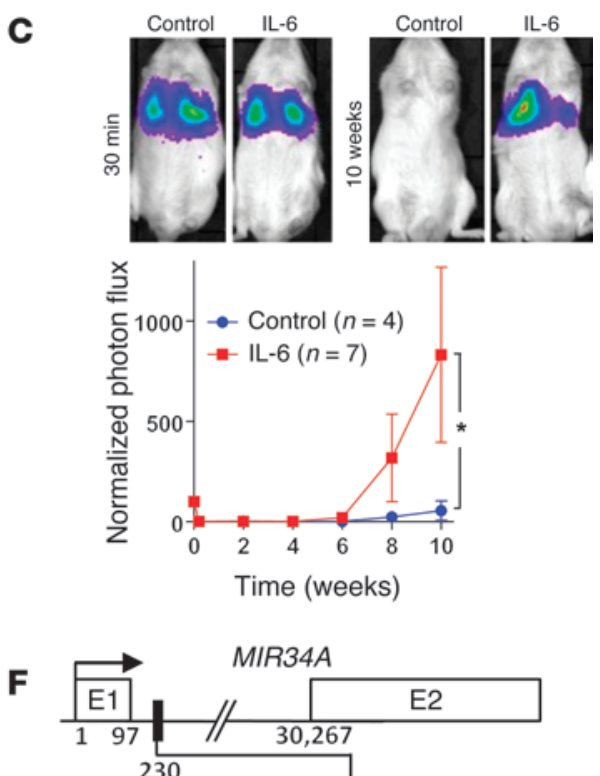

Human AAgGGGAAGAATTTCCAAGAAGCAACAAAATGT

Macaca AAGGGGAAGACTTTCCAAGAAGCAACAAAATGT

Mouse AAGGCGAAGAATTTCCAAGAAGTAACAAAATGT

Rat AAGGCGAAGAATTTCCAAGAAGTAACAAAATGT

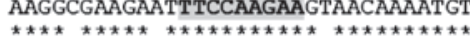

G

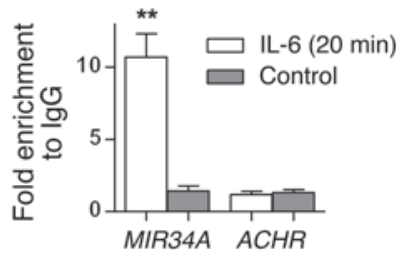

J DLD-1/pRTR-miR-34a

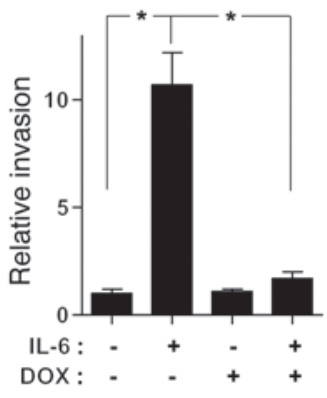

\section{Figure 1}

IL-6 induces EMT and invasion of CRC cells through direct repression of MIR34A by STAT3. (A) qPCR analysis of indicated mRNAs in DLD-1 cells treated with IL-6 for 5 days. (B) Relative invasion of DLD-1 cells transfected with indicated siRNAs for 24 hours, followed by IL-6 treatment for 72 hours. (C) Formation of lung metastases by tail-vein injection of control and IL-6-treated (5 days) DLD-1-Luc2 cells in immunocompromised mice. Representative images of luciferase signals (upper panel). Normalized photon flux (lower panel). (D) qPCR analysis of primary (pri-miR34a) and mature (miR-34a) miR-34a expression in DLD-1 cells treated with IL-6. (E) Expression of mature miR-34a in HT-29 CRC and MCF7 $\mathrm{BC}$ cells after treatment with IL-6 for 72 hours. (F) Map of the human MIR34A genomic region with the indicated phylogenetically conserved STAT3-binding site. (G) ChIP analysis of STAT3 occupancy at the human MIR34A and, as a control, the acetylcholine receptor (ACHR) locus in DLD-1 cells treated with vehicle or IL-6. (H) qPCR analysis of primary miR-34a in DLD-1 cells transfected with control or STAT3 siRNAs for 24 hours and subsequently treated with IL-6 for 72 hours. (I) qPCR analysis of indicated mRNAs in indicated cells, treated with IL-6 and DOX for 5 days. (J) Relative invasion of indicated cells treated with DOX for 24 hours and subsequently with IL-6 for 72 hours. Mean values \pm SD $(n=3)$ are provided. ${ }^{*} P<0.05 ;{ }^{*} P<0.01$. 

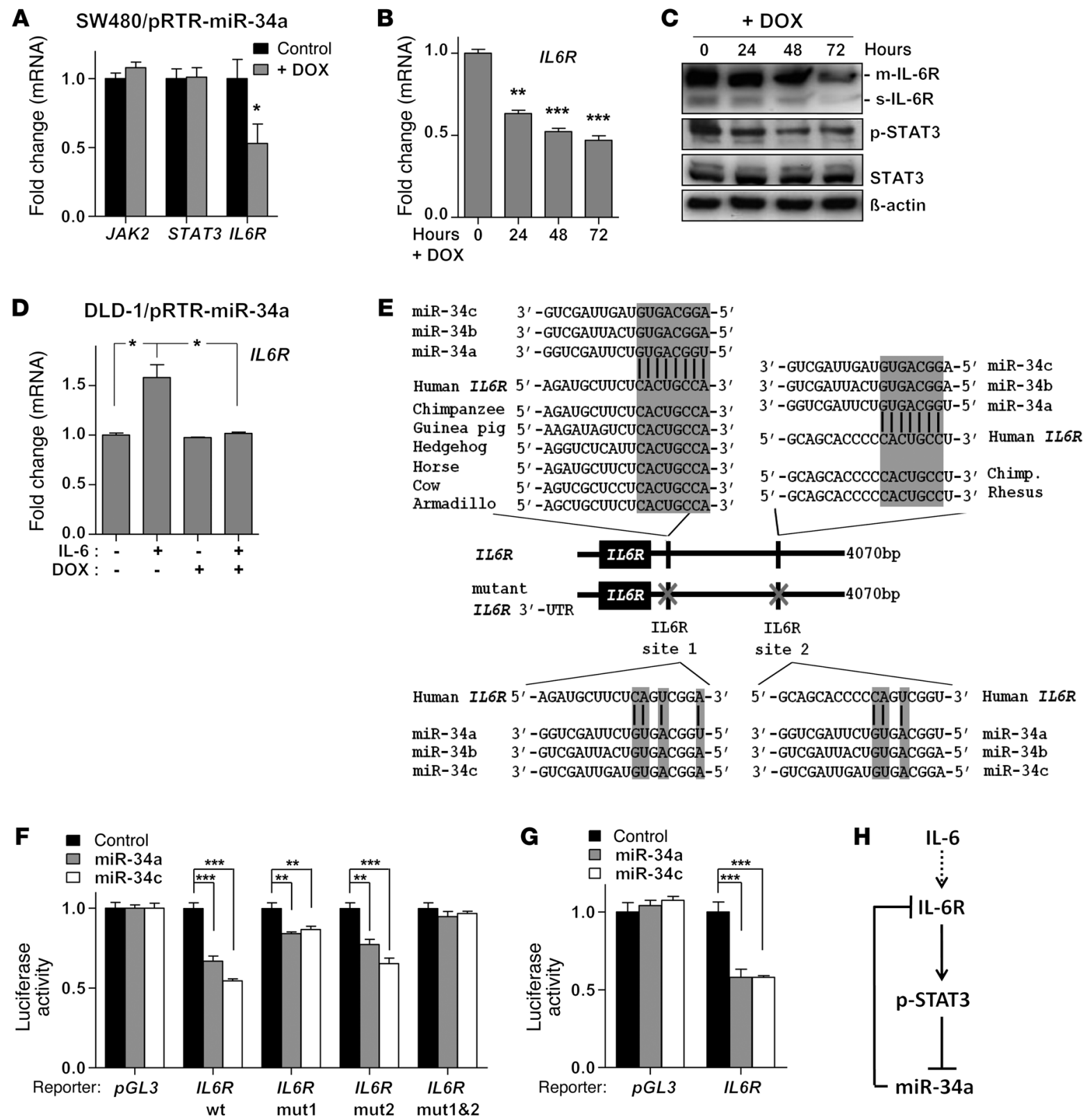

Figure 2

The IL6R mRNA is a direct target of miR-34. (A) qPCR analysis of the indicated mRNAs 48 hours after addition of DOX. (B) qPCR analysis of IL6R mRNA expression after addition of DOX for indicated periods. (C) Western blot analysis of the indicated proteins after addition of DOX for indicated periods. (D) qPCR analysis of IL6R mRNA expression in DLD-1/pRTR-miR-34a cells after IL-6 and DOX treatment for 5 days. (E) Schematic representation of the IL6R 3' UTR indicating the miR-34 seed-matching sequences, their phylogenetic conservation (upper part), and mutagenesis (lower part). (F and G) Dual reporter assay after transfection of $\mathrm{H} 1299$ cells with the indicated miRNA oligonucleotides with either (F) human or (G) murine IL6R 3' UTR reporter constructs. (H) Schematic representation of the proposed IL-6R/STAT3/miR-34a feedback loop. Mean values $\pm \mathrm{SD}(n=3)$ are provided. ${ }^{*} P<0.05$; ${ }^{* \star} P<0.01$; ${ }^{* \star *} P<0.001$.

after DOX treatment (Supplemental Figure 3A). Notably, the amount of phosphorylated STAT3 (p-STAT3) was also diminished by ectopic miR-34a expression (Figure 2C). In DLD-1 cells, IL6R expression increased after IL-6 treatment. However, this induction was prevented by ectopic miR-34a expression, demonstrating that it requires and is presumably mediated by repression of MIR34A (Figure 2D). In line with these results, we identified 2 phylogenetically conserved miR-34a seed-matching sequences in human IL6R
3' UTR (Figure 2E). The murine IL6R 3' UTR contains an additional miR-34a seed-matching sequence (Supplemental Figure 3B). Ectopic miR-34a and miR-34c repressed the activity of human and mouse IL6R 3' UTR reporter constructs in dual luciferase reporter assays (Figure 2, F and G). Mutation in either miR-34a-binding site partially abrogated this repression and mutations in both sites completely abrogated it (Figure 2F). Taken together, we showed that the IL6R mRNA is directly regulated by miR-34 via conserved 
A

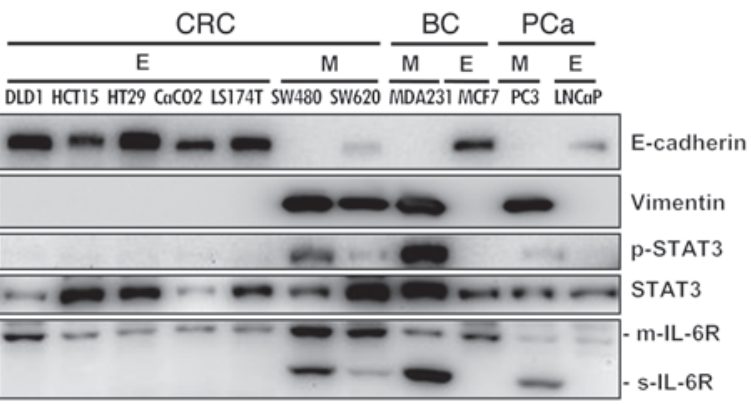

$\begin{array}{llllllllllll}1.00 & 0.47 & 0.41 & 0.48 & 0.69 & 2.97 & 1.58 & 0.77 & 0.88 & 0.41 & 0.57 & \mathrm{~m}-\mathrm{IL}-6 \mathrm{R} / \mathrm{a}-\mathrm{tub}\end{array}$

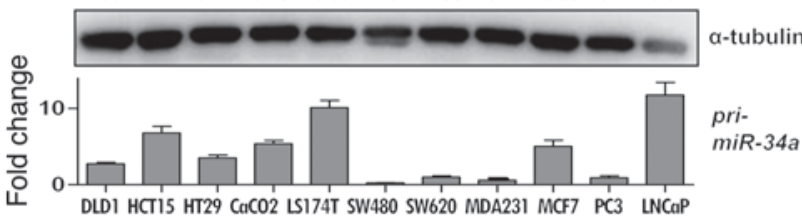

C

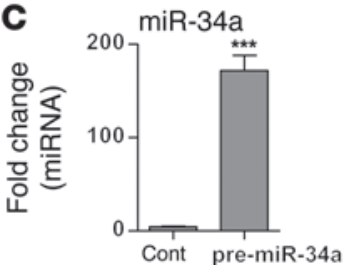

D

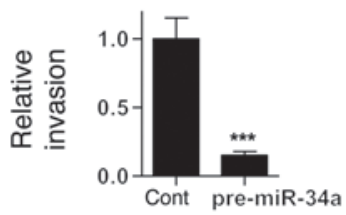

E
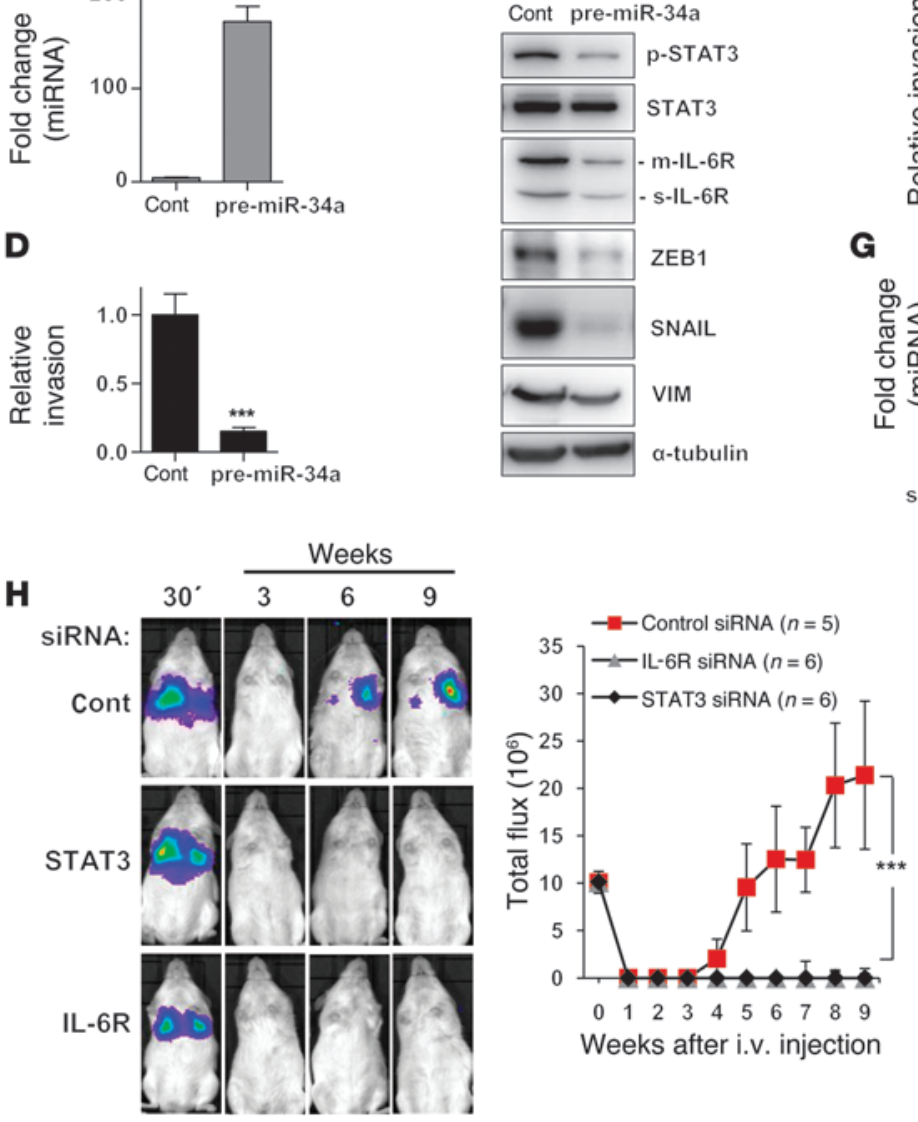

B SW480

Cont STAT3 IL-6R SIRNA
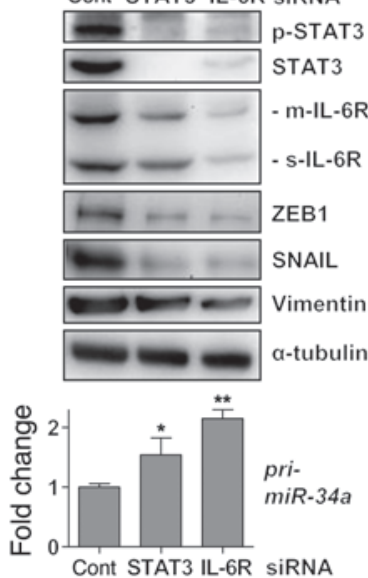

$\mathbf{F}$

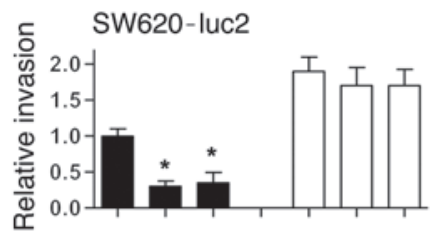

G

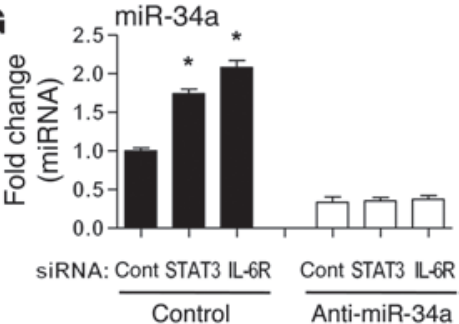

I



Figure 3

The mesenchymal phenotype of cancer cell lines is associated with the IL-6R/STAT3/ miR-34a loop. (A) Expression of the indicated proteins and primary miRNAs in CRC, breast cancer $(\mathrm{BC})$, and prostate cancer $(\mathrm{PCa})$ cell lines exhibiting epithelial (E) or mesenchymal $(\mathrm{M})$ phenotypes was determined by Western blot (upper panel) and qPCR analysis (lower panel). Relative densitometric quantifications of $\mathrm{m}-\mathrm{IL}-6 \mathrm{R}$ protein normalized to $\alpha$-tubulin are indicated. (B) Detection of protein and RNA expression 48 hours after transfection with the indicated siRNAs. (C, D, and E) Expression of miR-34a (C), invasion (D), and expression of indicated proteins (E) in SW480 cells transfected with control or pre-miR-34a oligonucleotides for 48 hours. (F) Relative invasion of SW620-luc2 cells transfected with STAT3or IL-6R-specific siRNAs and miR-34a-specific antagomirs. (G) qPCR analysis of mature miR-34a in cells treated as in F. (H and I) SW620-luc2 cells were transfected with control, STAT3-, or IL-6R-specific siRNAs for 48 hours, subsequently injected into the tail vein of NOD/SCID mice, and followed by noninvasive bioluminescence imaging for 9 weeks. In $\mathbf{H}$, the left panels show representative images and the graph shows the photon flux (right). Quantification of metastatic tumor nodules in the lung per mouse 9 weeks after tail-vein injection are shown (I). Mean values \pm SD $(n=3)$ are provided. ${ }^{*} P<0.05$; ${ }^{\star \star} P<0.01 ;{ }^{* \star} P<0.001$. seed-matching sequences. These results suggest the existence of a feedback loop that is activated by IL-6-mediated repression of MIR34A and subsequent activation of IL-6R and STAT3, which maintains the repression of MIR34A (Figure $2 \mathrm{H}$ ).

The IL-6R/STAT3/miR-34a loop is manifest in carcinoma lines with mesenchymal characteristics. To further examine whether activation of the IL-6R/STAT3/miR-34a feedback loop is a general feature of cancer cells exhibiting a mesenchymal phenotype, we compared established CRC and breast and prostate cancer cell lines known to display either epithelial or mesenchymal traits
(Figure 3A). We verified the reported epithelial or mesenchymal phenotype by detection of E-cadherin and Vimentin expression. Interestingly, the mesenchymal-like CRC lines SW 480 and SW620 showed a more pronounced expression of $\mathrm{m}-\mathrm{IL}-6 \mathrm{R}$ and s-IL-6R when compared with 5 CRC lines with epithelial traits. Also the breast and prostate cancer cell lines with mesenchymal traits expressed more IL-6R than the respective epithelial-like lines, but the increase was restricted to s-IL-6R. Furthermore, the mesenchymal lines displayed STAT3 phosphorylation, which was not detectable in the epithelial lines. Moreover, the 
A SW480/pRTR-p53

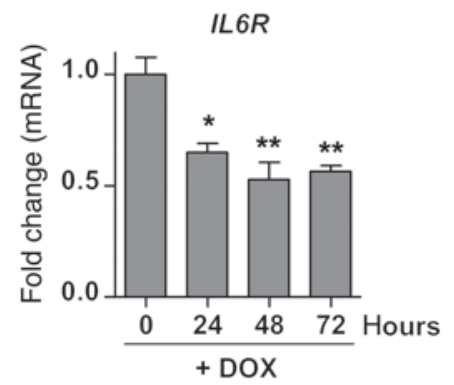

B SW480/pRTR-p53

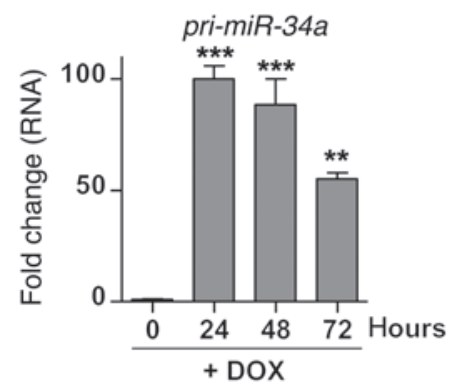

C DLD-1/tTA-p53

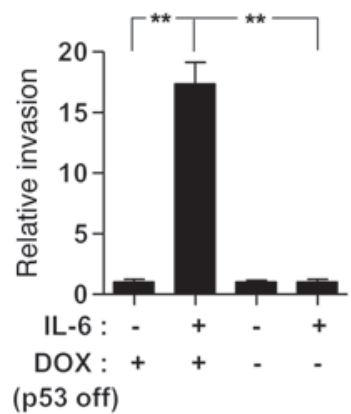

D

DLD-1/tTA-p53
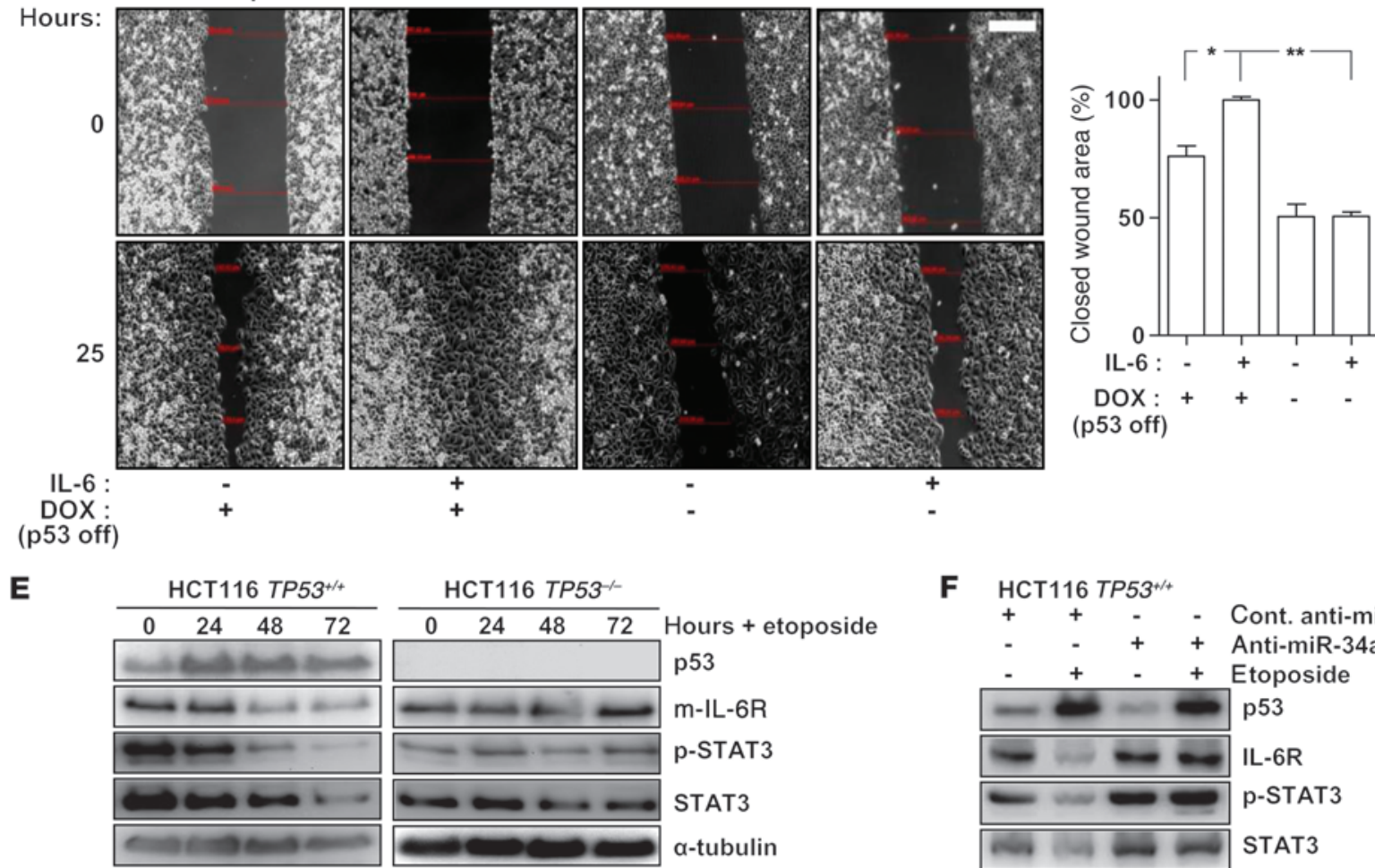

IL-6 :

p53 off

Hours + etoposide
p53
m-IL-6R
p-STAT3
STAT3
a-tubulin

$\mathbf{F}$

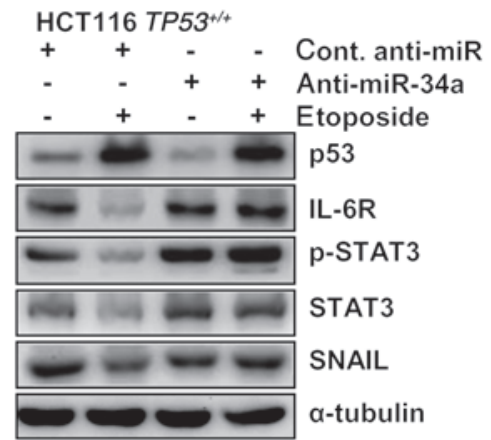

\section{Figure 4}

p53 disrupts the IL-6R/STAT3/miR-34a feedback loop by inducing miR-34a. (A) qPCR analysis of IL6R expression after addition of DOX for indicated periods. (B) qPCR analysis of primary miR-34a expression after addition of DOX for indicated periods. (C) Invasion assay in a modified Boyden chamber. DLD-1/tTA-p53 cells were depleted of DOX for 24 hours to induce ectopic p53, subsequently treated with IL-6 for 72 hours, and then allowed to migrate through Matrigel-coated filter for 48 hours. (D) Wound healing assay: DLD-1/tTA-p53 cells were depleted of DOX for 24 hours and subsequently treated with IL-6 for 72 hours before a scratch in the monolayer of cells was generated. Representative photographs of the initial wound area and the same area 25 hours later are provided in left panel. Twenty-five hours after a scratch was generated, the width of 5 scratches in 2 independent wells was analyzed for each state. Results represent the average (\%) of wound closure (right panel). Scale bar: $200 \mu \mathrm{m}$. (E) Western blot analysis of the indicated proteins in HCT116 TP53 ${ }^{+/+}$and HCT116 TP53 ${ }^{-/-}$cells after addition of etoposide (20 $\left.\mu \mathrm{M}\right)$ for indicated time periods. (F) Western blot analysis of HCT116 cells transfected with control or miR-34a-specific antagomirs for 24 hours, followed by addition of etoposide $(20 \mu \mathrm{M})$ for 48 hours. Mean values \pm SD $(n=3)$ are provided. ${ }^{*} P<0.05 ;{ }^{* \star} P<0.01 ;{ }^{* *} P<0.001$.

expression of mir-34a was markedly decreased in cell lines with mesenchymal traits when compared with those with epithelial features. In SW480 cells, which display high levels of p-STAT3 and IL-6R, downregulation of either STAT3 or IL-6R by siRNAs resulted in a decrease of p-STAT3, STAT3, and IL-6R levels, induction of mir-34a expression, and downregulation of the miR-34a target SNAIL as well as other mesenchymal marker proteins, such as Vimentin and ZEB1 (Figure 3B). Likewise, ectopic miR-34a expression (Figure 3C) decreased the invasion of SW480 cells (Figure 3D) and resulted in a decrease of 

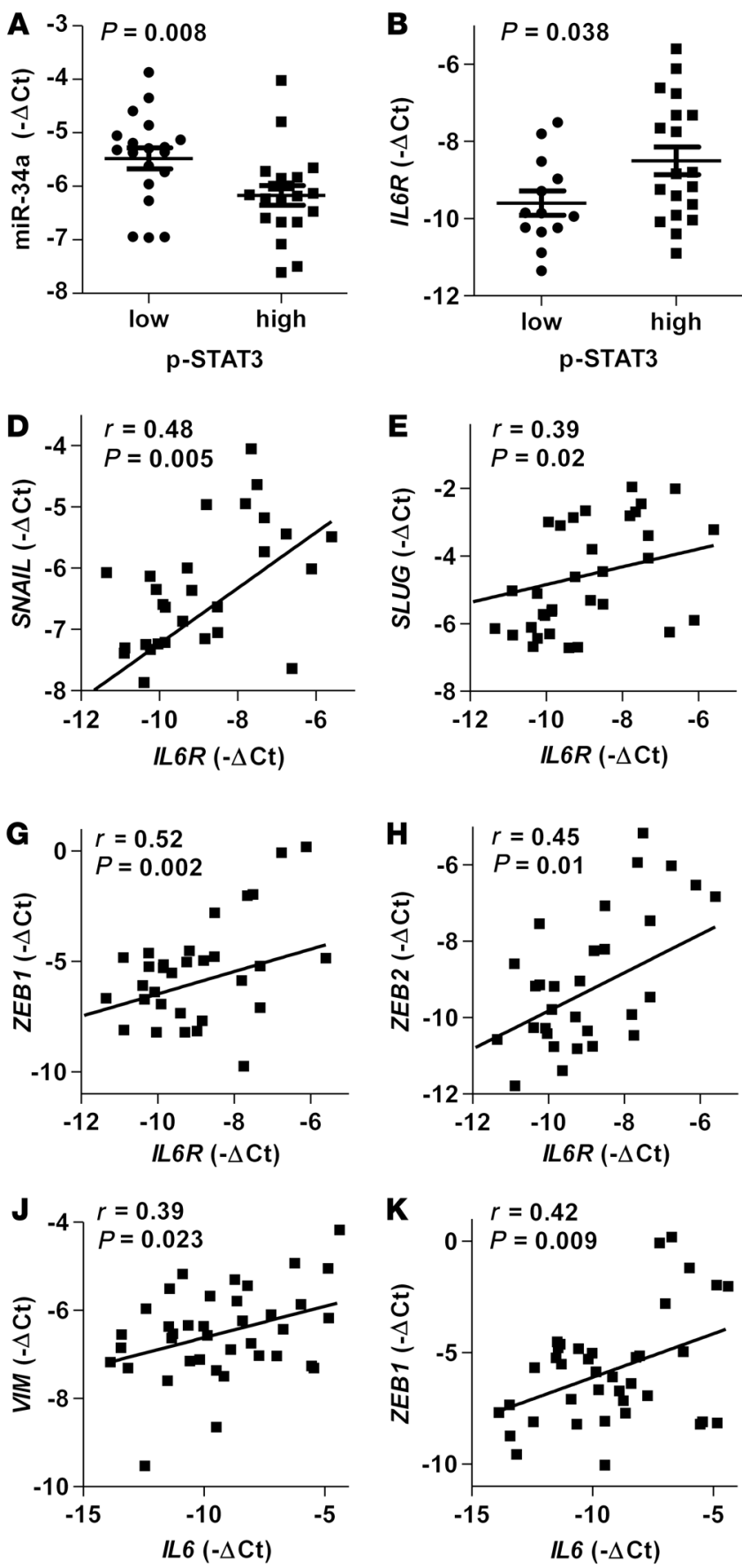

p-STAT3, IL-6R, ZEB1, SNAIL, and VIM expression (Figure 3E). Therefore, an active IL-6R/STAT3/miR-34a feedback loop is not only characteristic but also required for the maintenance of the mesenchymal phenotype in CRC lines.

Role of the IL-6R/STAT3/miR-34 a feedback loop in invasion and metastasis. Previously, we have shown that ectopic expression of miR$34 \mathrm{a}$ in SW480 cells leads to MET accompanied by decreased invasion (18). Here, knockdown of STAT3 or IL-6R also resulted in decreased invasion of SW620-luc2 and SW480 cells, which was prevented by antagomir-mediated inactivation of miR-34a, indicating that repression of MIR34A by STAT3 is required for cellular invasiveness (Figure 3, F and G, and Supplemental Figure 4A).
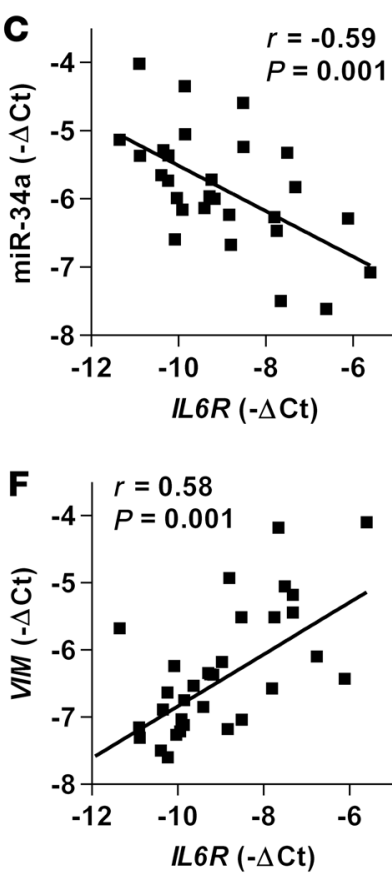

Figure 5

The IL-6R/STAT3/miR-34a feedback loop is characteristic for primary $\mathrm{CRC}$ tumors with mesenchymal features. (A-L) Correlative analysis of the indicated mRNAs, miRNAs, and p-STAT3 in human colon tumors $(n=48)$. p-STAT3 was detected by immunohistochemistry, while expression of mRNAs and mature miR-34a was determined by qPCR. Mean values \pm SEM are provided (A and B). Significance was calculated using the Student's $t$ test (A and B). Spearman correlation coefficient with the respective significance is indicated (C-L).
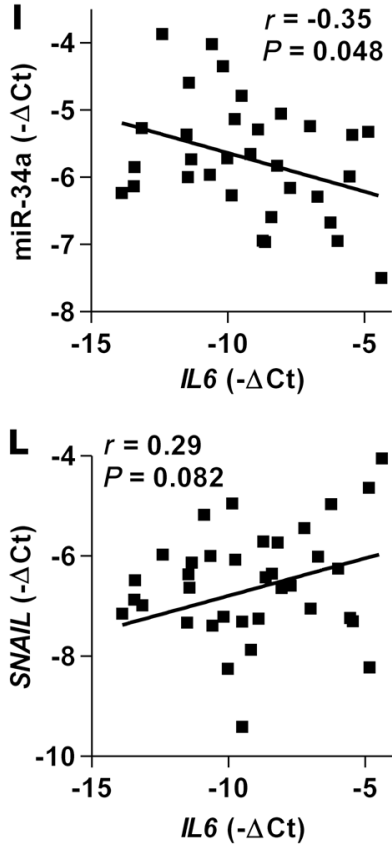

Since SW620 cells express some miR-34a, the antagomir-mediated repression of miR-34a in SW620 cells resulted in increased invasion, presumably since these cells still express a minor amount of miR-34a (Figure 3, A and F). However, expression of miR-34a in SW480 cells is very low or absent (Figure 3A), explaining why miR-34a antagomirs did not affect invasion in these cells (Supplemental Figure 4A). Finally, downregulation of STAT3 or IL-6R by siRNAs in SW620-luc2 cells suppressed metastasis formation in NOD/SCID mice after tail-vein injection (Figure $3 \mathrm{H}$ and Supplemental Figure 4B) and reduced the number of metastatic tumor nodules in the lungs of mice 9 weeks after tail-vein injection (Figure 3I and Supplemental Figure 4, C and D). Taken together, 

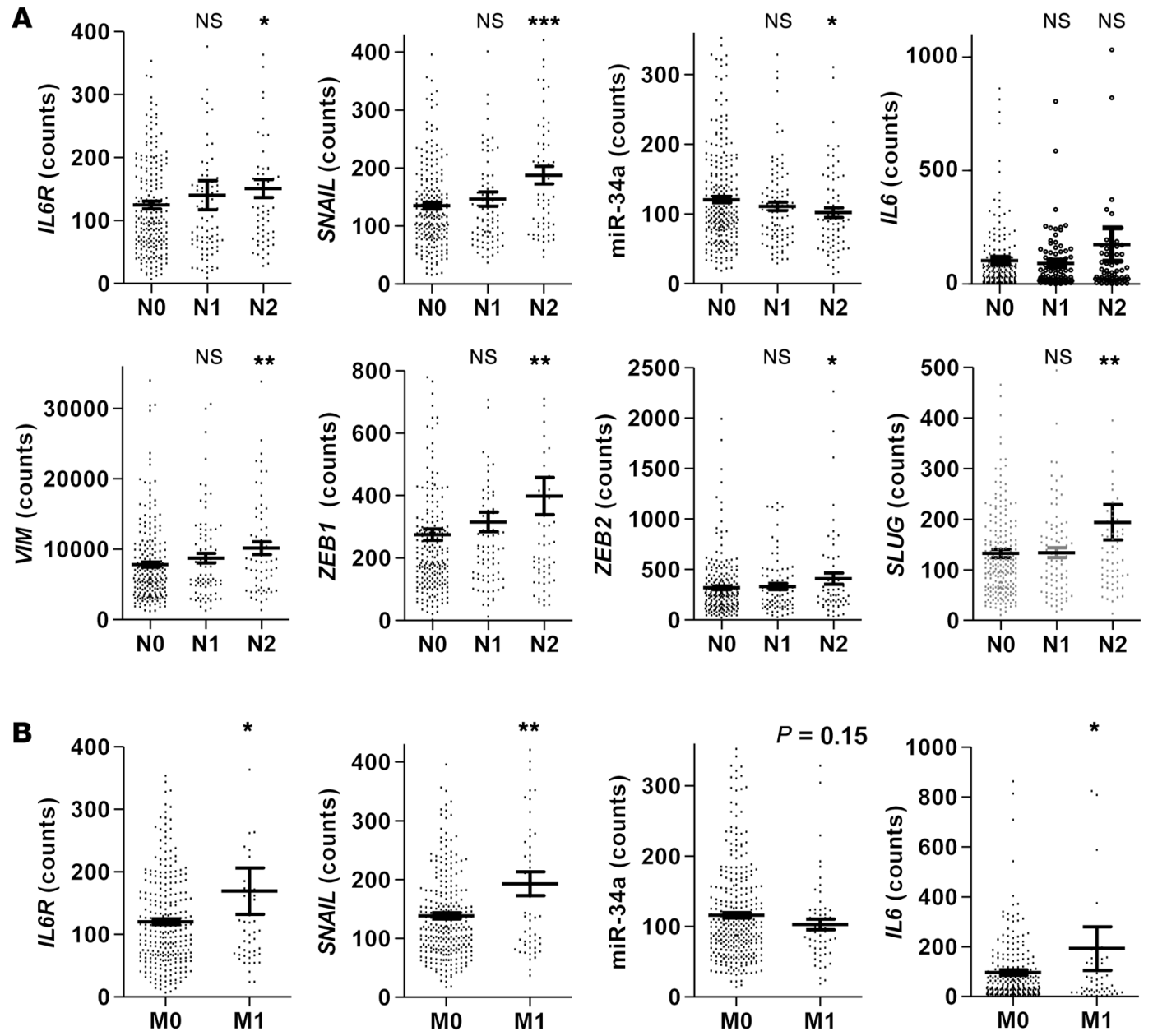

Figure 6

Activation of the IL-6R/STAT3/miR-34a feedback loop is associated with lymph node and distant CRC metastasis. (A) Associations of indicated genes with nodal status in the TCGA collection of human colon adenocarcinomas $(n=425)$. (B) Associations of indicated genes with metastasis status in the TCGA collection of human colon adenocarcinomas. Mean values \pm SEM are provided. Significance was calculated using Student's $t$ test. ${ }^{*} P<0.05 ;{ }^{* *} P<0.01 ;{ }^{* * *} P<0.001$.

these results imply that the IL-6R/STAT3/miR-34a feedback loop is necessary for invasion and metastasis formation of CRC cells, which had undergone an EMT. Since p53 is mutated in DLD-1, SW480, and SW620 cells, the regulation and biological effects of the IL-6R/STAT3/miR-34a loop in these cells are presumably not mediated by $\mathrm{p} 53$.

p53 disrupts the IL-6R/STAT3/miR-34a feedback loop by inducing miR-34a. p53 activation has been shown to induce MET via directly inducing the members of the $m i R-200$ and $m i R-34$ families (summarized in ref. 16). Therefore, we hypothesized that the downregulation of IL6R expression by miR-34a may contribute to MET mediated by p53. Indeed, ectopic expression of p53 in SW480 cells resulted in a pronounced repression of IL6R mRNA, which was accompanied by induction of miR34a expression (Figure 4, A and B, and Supplemental Figure 5A). Ectopic expression of $\mathrm{p} 53$ in DLD-1 cells prevented invasion induced by treatment with IL-6 (Figure 4C and Supplemental Figure 5B). Moreover, ectopic expression of p53 also prevented IL-6-induced migration (Figure 4D). Furthermore, treatment with the DNA-damaging agent etoposide resulted in the downregulation of IL-6R expression and decreased STAT3 phosphorylation in HCT116 $\mathrm{TP} 3^{+/+}$, but not in isogenic HCT116 TP53-/- cells (Figure 4E), indicating that this effect was p53 dependent. We also observed a minor downregulation of STAT3 expression in $\mathrm{TP} 53^{+/+}$cells. A similar observation was reported previously (31). When miR34 a was specifically inhibited by an antagomir, the repression of IL-6R and STAT3 as well as the p53-mediated decrease in STAT3 phosphorylation was prevented (Figure 4F and Supplemental Figure 5C), demonstrating that miR-34a mediates these effects of $\mathrm{p} 53$. The previously reported miR-34a-mediated downregulation of SNAIL after p53 activation (18) served as a control. Finally, treatment of the p53 WT cell line LoVo with IL-6 resulted in reduction of miR-34a expression; however, additional activation of p53 with etoposide counteracted this effect (Supplemental Figure 5D). The expression of the STAT3 target gene BLC3 increased after IL-6 treatment, which shows that LoVo cells are responsive to IL-6 (Supplemental Figure 5D). Taken together, activation of p53 disrupts the IL-6R/STAT3/miR-34a feedback loop via the induction of miR-34a, which may thereby critically contribute to tumor suppression by p53. 
The IL-6R/STAT3/miR-34 a feedback loop is characteristic for primary CRC tumors with mesenchymal features. To test whether the regulations described above for CRC cell lines are also clinically relevant, we examined primary CRC specimens derived from 48 CRC patients. Indeed, we could detect a negative correlation between p-STAT3 levels and the expression of miR-34a (Figure 5A) and a positive association of P-STAT3 and IL6R expression (Figure $5 \mathrm{~B})$. Moreover, expression of miR-34a negatively correlated with IL6R (Figure 5C), whereas expression of IL6R positively correlated with EMT-associated mesenchymal makers SNAIL, SLUG, VIM, ZEB1, and ZEB2 (Figure 5, D-H). Likewise, the expression of IL6 negatively correlated with miR-34a (Figure 5I) and positively with mesenchymal markers VIM, ZEB1, SNAIL, and ZEB2 (Figure 5, J-L and Supplemental Figure 6). We also observed a significant negative correlation between SLUG and miR-34a and a negative correlation of SNAIL and miR-34a, which was, however, not statistically significant (Supplemental Figure 6, A and B).

Additionally, we analyzed data from 425 patients with colorectal adenocarcinoma deposited in the Cancer Genome Atlas (TCGA) database (Cancer Genome Atlas Network; ref. 32). Primary CRCs of patients with nodal status pN2 displayed significantly elevated expression of IL6R, SNAIL, VIM, ZEB1, ZEB2, and SLUG and decreased levels of miR-34a when compared with patients with nodal status pN0 (Figure 6A). Moreover, primary tumors, which displayed distant metastases (M1), showed a significant association with increased expression of IL6R, IL6, and SNAIL (Figure 6B). Furthermore, primary M1 tumors displayed decreased levels of miR-34a expression (Figure 6B). However, this difference rather represented a trend, since it was not statistically significant. In summary, these results show that the IL-6R/ $\mathrm{STAT} 3 / \mathrm{miR}-34$ a loop is also active in primary human colon cancers and contributes to a mesenchymal phenotype of tumor cells, which promotes invasion and metastasis.

Deletion of Mir34 a facilitates tumor invasion in a mouse model of colitis-associated cancer. In order to analyze the relevance of an IL-6R/ STAT3/miR-34a feedback loop in an autochthonous colorectal tumor model we employed the well-established azoxymethane/ dextran sodium sulfate (AOM/DSS) model (33), which closely recapitulates human colitis-associated cancer (CAC). AOM exposure results in mutations in the $\beta$-catenin/Ctnnb that initiate colorectal carcinogenesis (34). Repeated DSS administration causes chronic inflammation, which greatly enhances the incidence of AOM-induced tumors (35). Since sporadic intestinal tumors are also characterized by an inflammatory microenvironment (9), this mouse model also recapitulates many aspects of sporadic intestinal cancers. Since miR-34a was expressed at more than approximately 1000-fold higher levels than miR-34b and miR-34c in intestinal epithelia and approximately 180-fold higher in intestinal tumors (data not shown), we focused on the role of miR-34a in CAC. Compared with that in WT mice, expression of miR-34a was markedly elevated in colon tissue of mice with intestinal cell-specific STAT3 deletion (Stat $3^{\triangle I E C}$ ) (Figure 7A). DSS treatment of Mir $34 a^{F / F}$ mice for 5 days resulted in decreased expression of miR-34a in colon epithelial cells accompanied by an induction of IL6 expression in colon tissue (Figure 7, B and C). In line with the results described above, Mir34a is therefore also repressed in vivo by inflammatory signaling known to involve STAT3 activation (36). Next, we focused on the role of miR-34a in AOM/DSS-induced carcinogenesis using Mir34a-deficient mice we had recently generated (Figure 7, D-F, and Supplemen- tal Figure 7). So far, Mir34 $4 a^{-/-}$mice did not display an obvious phenotype nor an increase in spontaneous tumor formation and showed normal Mendelian inheritance (data not shown). However, upon AOM/DSS challenge, the incidence and size of tumors were significantly enhanced in Mir $34 a^{-/-}$mice (Figure 7, G and H). Furthermore, Mir34a-deficient tumors revealed increased cell proliferation and decreased apoptosis when compared with Mir34a-proficient tumors (Figure 7, I and J). Importantly, approximately $70 \%$ of $\mathrm{Mir} 34 \mathrm{a}^{-/-}$mice displayed invasive tumors that penetrated through the muscularis mucosa, whereas none of the tumors from Mir $34 a^{F / F}$ mice showed invasion (Figure 7, $\mathrm{K}$ and $\mathrm{L}$ ). Loss of p53 has been suggested to be essential for colonic tumor invasion (37) and was recently linked to activation of NF- $\kappa \mathrm{B}$ and Stat3 (38), which also comprise important signaling nodes in CAC $(39,40)$. However, sequencing the entire p53 coding region of tumor cells from $6 \mathrm{Mir} 34 \mathrm{a}^{F / F}$ and $10 \mathrm{Mir} 34 \mathrm{a}^{-/-}$ mice did not reveal any mutations in either genotype. Therefore, the deletion of miR-34a presumably substituted for the inactivation of p53 as a requirement for invasiveness.

Similarly to human IL6R, the murine Il6r 3' UTR was also repressed by miR-34 through conserved mir-34 seed-matching sequences (Figure 2, F and G). Moreover, in the murine rectal cancer cell line CMT93, which expresses constitutively active p-STAT3, siRNA-mediated downregulation of STAT3 resulted in increased miR-34a expression (Figure 8A). Furthermore, immunohistochemical analyses showed increased phosphorylation of STAT3 and elevated expression of IL-6R in tumor cells of Mir34a $a^{-/-}$mice compared with Mir34 $a^{F / F}$ mice (Figure 8, $\mathrm{B}$ and C). Also, the levels of the EMT inducer and miR-34 target SNAIL were markedly elevated in $\mathrm{Mir} 34 \mathrm{a}^{-/-}$mice, suggesting that tumor cells in these mice underwent EMT (Figure 8, B and C). Finally, Mir $34 a^{-/-}$tumors displayed increased STAT3 phosphorylation and expressed higher levels of IL-6R, SNAIL, and ZEB1 protein and RNA when compared with Mir34 $a^{F / F}$ tumors (Figure 8, D and E). Taken together, miR-34a loss activates IL-6R/STAT3 signaling in colitis-associated tumors and allows invasion. In combination with the functional analysis of human CRC cells described above, the activation of the IL-6R/ STAT3/miR-34a circuitry promotes EMT and invasion and may ultimately lead to metastases.

\section{Discussion}

Here, we identified an IL-6-triggered feedback loop that involves STAT3-mediated repression of MIR34A and controls EMT and colonic tumor progression. This study also provides what we believe is the first in vivo proof for a tumor-suppressive function of Mir34a in a genetic mouse model. Our analysis revealed the presence of the IL-6R/STAT3/miR-34a loop in all analyzed carcinoma cells with mesenchymal and therefore metastatic traits, implying that it may represent a new unifying mechanism of carcinogenesis. Nonetheless, tissue and celltype-specific differences in the activation of the IL-6R/STAT3/ miR-34a loop may exist, since STAT3 activation can be achieved via multiple other signaling pathways, such as receptor-tyrosine kinases, deregulated in cancer (41). Recent observations suggest that during metastasis, tumor cells acquire a mesenchymal phenotype, which allows them to leave the site of the primary tumor, invade surrounding tissues, and migrate to distant organs, whereas after seeding, these cells switch back to an epithelial phenotype to efficiently proliferate and form metasta- 

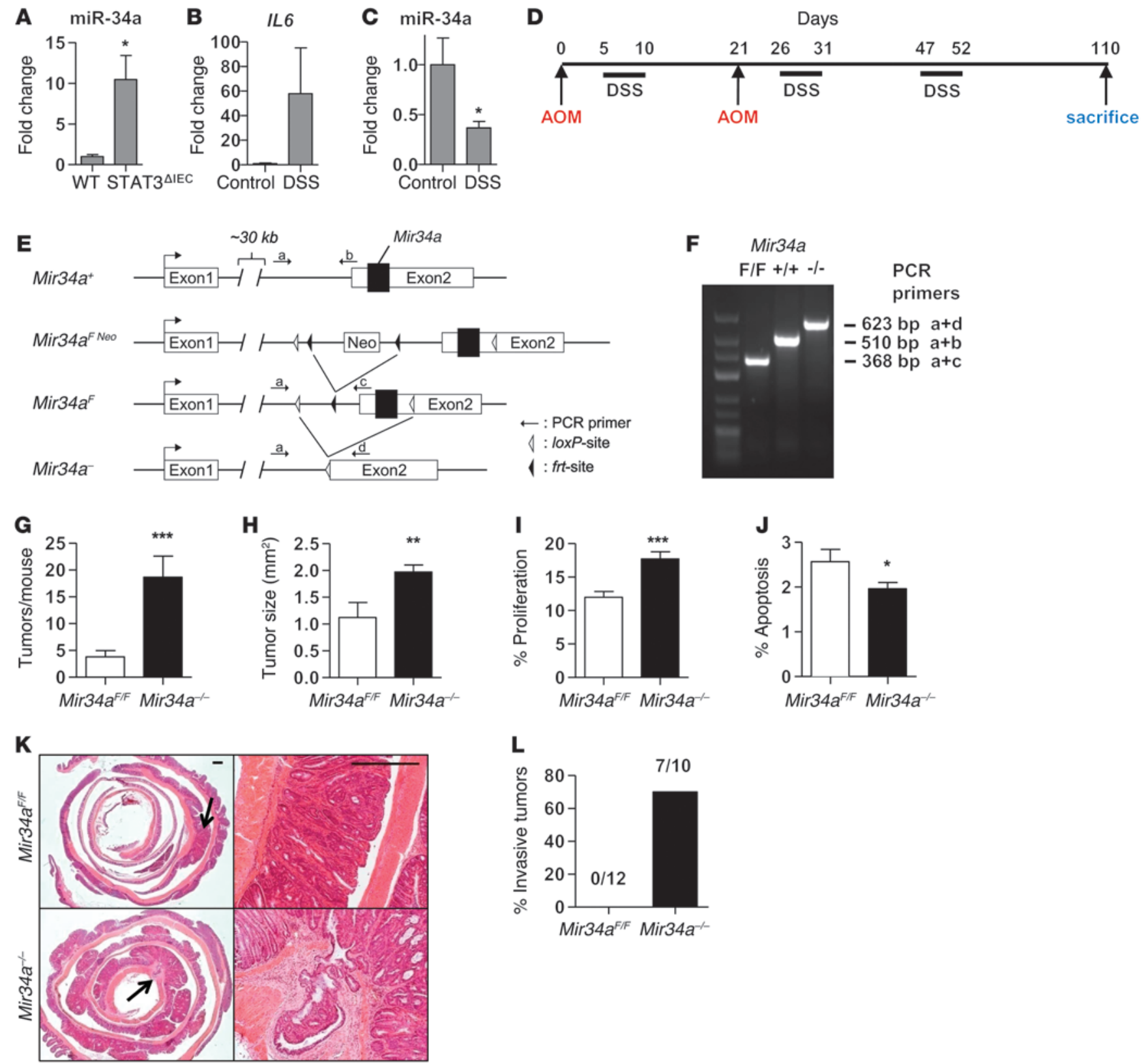

\section{Figure 7}

Loss of MiR34a facilitates tumor invasion after AOM/DSS treatment. (A) Expression of miR-34a in colon tissue of WT and Stat3 ${ }^{\Delta I E C}$ mice. (B) Expression of $/ / 6$ mRNA in colon tissue of Mir34a F/F mice after 5 days of DSS treatment. (C) Expression of miR-34a in colon epithelial cells of Mir34aF/F mice after 5 days of DSS treatment. (D) Schematic overview of the CAC regimen as described in Methods. (E) Outline of the Mir34a targeting strategy in mice. (F) Genotyping PCR for determination of Mir34a- ${ }^{--}$mice. (G) Tumor incidence in indicated mice ( $n \geq 10$ for each genotype). (H) Mean tumor size in indicated mice ( $n \geq 5$ for each genotype). (I) Tumor cell proliferation was determined by BrdU incorporation. Percentage of proliferation indicates BrdU-positive cells ( $n \geq 10$ tumors of each genotype). (J) Tumor cell apoptosis was determined by detection of cleaved caspase-3. Percentage of positive cells is indicated ( $n \geq 10$ tumors of each genotype). (K) H\&E-stained sections of colons from Mir34a $a^{F / F}$ and Mir34a ${ }^{-/}$mice with arrows indicating magnified areas showing tumor morphology and representative invasive colon carcinoma in Mir34a-/- mice. Scale bars: $500 \mu \mathrm{m}$. (L) Percentage of mice showing invasive tumors for indicated genotypes. Number of mice with invasive tumors/total number of mice for each genotype is indicated above the bars. Mean values \pm SEM are provided. ${ }^{*} P<0.05 ;{ }^{* *} P<0.01 ;{ }^{* *} P<0.001$.

ses $(3,42)$. The activation of the IL-6R/STAT3/miR-34a loop by IL- 6 induces EMT and might shift the cellular phenotype toward a mesenchymal state that is advantageous for invasion, intravasation, and extravasation steps of the metastatic cascade. On the contrary, interference with the loop by p53 activa- tion induces MET and might allow cells to switch back to an epithelial state, which allows colonization and outgrowth of metastases (Figure 8F). The reversible character of the IL-6R/ STAT3/miR-34a loop promises to allow multiple therapeutic interventions aimed at blocking its prometastatic activity. 
A CMT93 cells
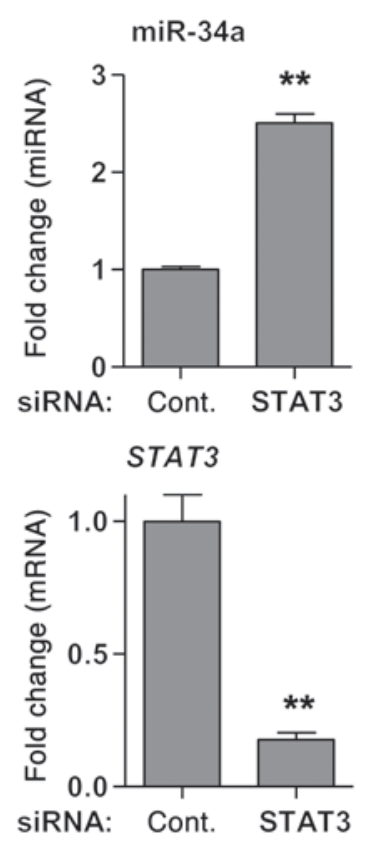

B

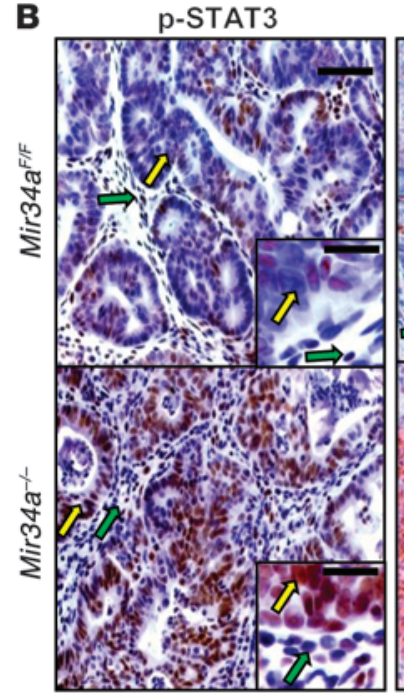

C





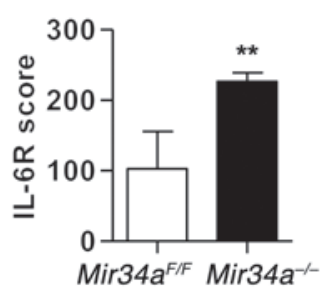

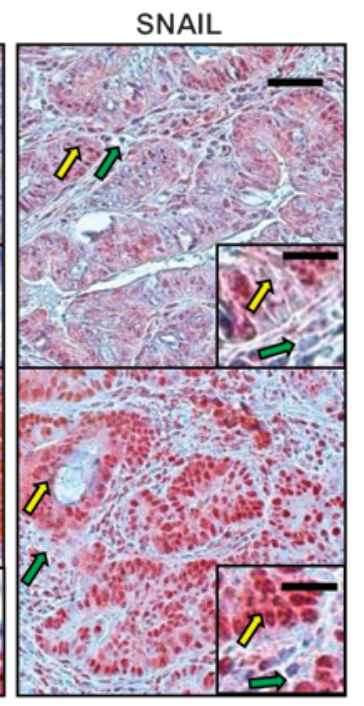

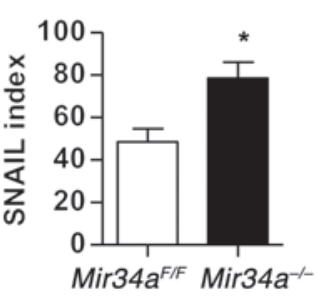

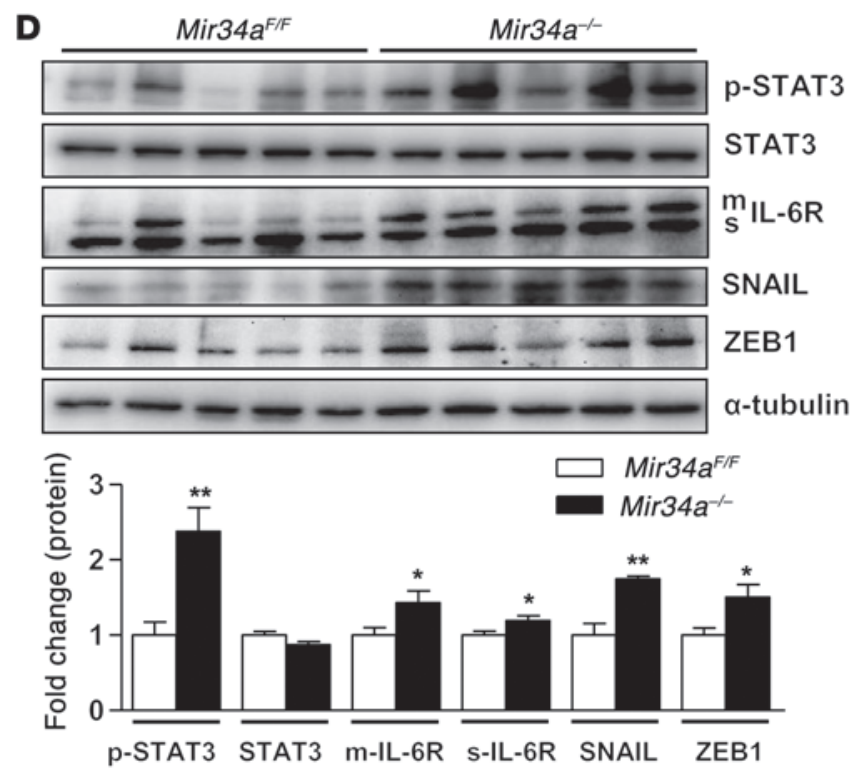

E

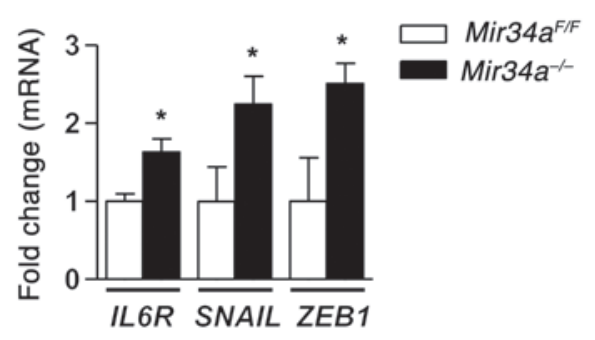

F

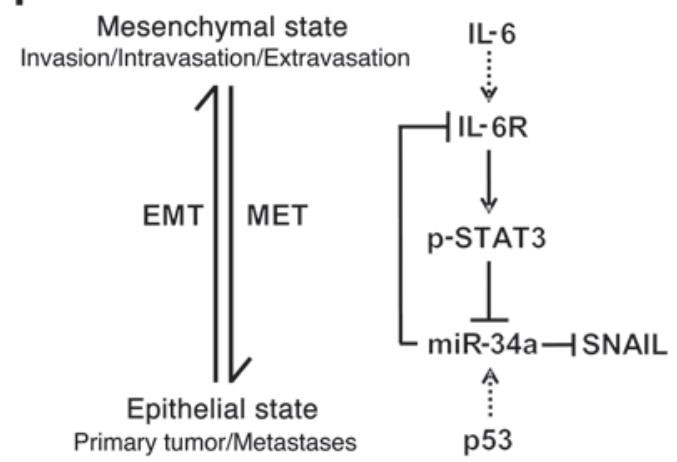

Figure 8

The tumors of Mir34a $\mathrm{a}^{-/}$mice show characteristics of EMT and enhanced IL-6R/STAT3 signaling. (A) Expression of mature miR-34a and Stat3 in mouse rectal cancer cell line CMT93 transfected with control or STAT3 siRNA. (B and C) Immunohistochemical analysis of p-STAT3, IL-6R, and SNAIL in tumors of Mir34a ${ }^{F / F}$ and Mir34a $a^{-/-}$mice. Yellow and green arrows indicate tumor and stromal cells, respectively. Scale bars: $50 \mu \mathrm{m}$; $20 \mu \mathrm{m}$ (insets). Bar charts (C) show the percentage of positive tumor cells (index; p-STAT3 and SNAIL) or expression score (IL-6R) determined as described in Methods ( $n \geq 10$ tumors from each genotype). (D) Western blot analysis of indicated proteins in lysates prepared from tumors of Mir34a $a^{F / F}$ and Mir34a $a^{--}$mice ( $n=5$ from each genotype). Relative densitometric quantifications of indicated proteins are shown in lower panel. (E) QPCR analysis of indicated mRNAs in tumors of Mir34a $\mathrm{F}^{\mathrm{FIF}}$ and Mir34a- mice ( $n=3$ from each genotype). (F) Schematic representation of the proposed IL-6R/STAT3/miR-34a feedback loop and its potential involvement in cancer progression. The activation of the loop by IL-6 induces EMT and shifts the cellular phenotype toward a mesenchymal state that is advantageous for invasion, intravasation, and extravasation steps of the metastatic cascade. On the contrary, the inactivation of the loop by p53 induces MET and switches cells to an epithelial state, which allows colonization and outgrowth of metastases. Shown are mean values \pm SEM. ${ }^{\star} P<0.05 ;{ }^{* \star} P<0.01 ;{ }^{* \star} P<0.001$. 
The AOM/DSS model recapitulates colitis-associated carcinogenesis, which was shown to require IL-6, STAT3, and the IL-6R dimerizing partner gp130 $(39,40)$, yet did not result in invasive tumors. Although in control Mir $34 a^{F / F}$ mice, DSS-induced colitis led to a marked suppression of miR-34a, this was not sufficient to drive tumor invasion. Supposedly, p53 activation, which is observed after DSS treatment (43), can counteract this repression by keeping Mir34a expression above a certain threshold. Indeed, miR-34a expression was decreased in invasive tumors of AOMchallenged Tp5 $3^{\triangle I E C}$ mice (S. Schwitalla and F.R. Greten, unpublished observations) in a model of sporadic CRC (10). Furthermore, these data suggest that the IL-6R/STAT3/miR-34a loop is also of relevance for CRC, which is further supported by our analysis of sporadic CRC patients and its requirement for the maintenance of the mesenchymal phenotype of invasive CRC cell lines. Moreover, because inactivation of MIR34A by CPG island methylation, deletion, or downregulation is commonly found in various human cancers (16), the IL-6R/STAT3/miR-34a loop might be involved not only in the progression of CRC, but also in other cancer types. Accordingly, we also detected activation of this loop in established breast and prostate cancer cell lines with mesenchymal traits.

While we show that IL-6 activates the IL-6R/STAT3/miR-34a loop, this circuit most likely can also be triggered by other extrinsic signaling from the tumor microenvironment or cancer cell intrinsic signaling (such as oncogene activation and tumor suppressor inactivation) that activates IL-6R or STAT3, or suppresses miR34a. Besides p53, miR-34a can also be directly induced by ELK-1 and repressed by HSF-1 $(44,45)$ and, as shown here, by STAT3. Once activated, the circuit can be permanently active through its positive feed-forward nature. While the constitutive activation of the components in the circuit is interdependent, each component regulates its own set of downstream genes that together drive cancer progression. Several known targets of the components of the IL-6R/STAT3/miR-34a loop might be important for cancer progression, e.g., STAT3 can directly induce the EMT activator ZEB1 (46). Furthermore, we and others have recently shown that SNAIL, another well-established inducer of EMT, is a direct target of miR-34a $(18,19)$. Here, SNAIL was induced after IL-6 treatment, repressed after STAT3 or IL-6R knockdown, and expressed at elevated levels in tumors of $\mathrm{MiR} 34 \mathrm{a}^{-/-}$mice. Others showed that SNAIL induces IL-6, thereby providing additional feedback that may reinforce the loop (47). Collectively, these results suggest that SNAIL is an important effector of the IL-6R/STAT3/miR-34a loop. This is supported by the notion that IL- 6 also promotes head and neck tumor metastasis and EMT via a STAT3/SNAIL signaling pathway (48). Furthermore, IL-6 promotes growth and invasion of breast cancer cells through STAT3-dependent upregulation of NOTCH signaling (49). Interestingly, NOTCH1 represents a miR$34 \mathrm{a}$ target (50). Among other direct miR-34a targets that induce cancer cell invasion are c-KIT (51), AXL (52), and c-MET (53). We recently showed that the zinc finger 281 protein (ZNF281) is also an important miR-34 target with respect to EMT (54). We demonstrated that the expression of ZNF281 is controlled by miR-34 and SNAIL in a coherent feed-forward loop, where SNAIL and ZNF281 induce each other, whereas miR-34 can directly repress both of them. Accordingly, ectopic ZNF281 induces EMT by directly activating SNAIL and mediates increased migration and invasion. Therefore, miR-34a might have tissue-specific effectors that function via an activated IL-6R/STAT3/miR-34a loop.
For activation of STAT3, the IL-6R requires the ligand IL-6, which allows binding to gp130. In vivo, IL- 6 can be generated by tumor cells or by tumor stromal cells, such as macrophages or fibroblasts (12). The RNA used for the expression analysis of human CRC samples was isolated from nonmicrodissected tumors. Therefore, several stromal cells could have contributed to IL-6 expression. However, the CRC cell lines with a mesenchymal phenotype, which consist only of tumor cells, showed elevated expression of the s-IL-6R, which is involved in IL-6 transsignaling (55) and is also under control of miR-34a. In the AOM/DSS CAC mouse model, expression of the m-IL-6R decreases, but sIL-6R expression increases during tumor development $(30,56)$. In Mir34a-deficient mice, the levels of the s-IL-6R were further increased, suggesting that inhibition of s-IL-6R by miR-34a suppresses tumor progression. The analysis of human CRC samples suggests that the IL-6R/STAT3/miR-34a loop is also manifest in primary human colorectal tumors with mesenchymal characteristics and might represent a useful prognostic marker for cancer progression. In line with these findings, we recently showed that the loss of miR-34a expression by epigenetic silencing in primary tumors is associated with increased lymph node infiltration and metastasis in colon cancer patients (57). Besides STAT3 and IL-6R, which are already established targets for cancer treatment, our results suggest that restoring $\mathrm{miR}-34$ a function using mimetics may have therapeutic potential for the treatment of invasive CRCs. Furthermore, recombinant, soluble gp130, which selectively inhibits the s-IL-6R (58), may be especially suitable for the treatment of invasive CRC displaying MIR34A inactivation and therefore upregulation of IL-6R expression.

\section{Methods}

Cell lines/culture and reagents. CRC (SW480, SW620, Caco-2), breast cancer (MCF7, MDA-MB-231), and prostate cancer (LNCaP, PC-3) cell lines were maintained in DMEM (Invitrogen) containing 10\% FBS (Invitrogen). The CRC cell lines HCT-15, HT29, LS174T, HCT116, and DLD-1 and its derivatives were maintained in McCoy's 5A Medium (Invitrogen) containing 10\% FBS. The HCT116 TP53-- and DLD-1/tTA-p53 cells were a gift from Bert Vogelstein (Johns Hopkins Medical School, Baltimore, Maryland, USA). All cells were cultivated in the presence of 100 units $/ \mathrm{ml}$ penicillin and $0.1 \mathrm{mg} / \mathrm{ml}$ streptomycin. Doxycycline (DOX) (Sigma-Aldrich) was dissolved in water $(100 \mu \mathrm{g} / \mathrm{ml}$ stock solution) and always used at a final concentration of $100 \mathrm{ng} / \mathrm{ml}$ unless otherwise indicated. IL-6 (Immunotools) was dissolved in water and used at a final concentration of $20 \mathrm{ng} / \mathrm{ml}$. Etoposide (Sigma-Aldrich) was used at a concentration of $20 \mu \mathrm{M}$. siRNAs (Ambion silencer siRNA: negative control [ID\#4611], STAT3 [ID\#6880], and IL-6R [ID\#8642]) were transfected at a final concentration of $10 \mathrm{nM}$ using HiPerfect transfection reagent (QIAGEN).

Generation of cell pools stably expressing conditional alleles. Stable DLD-1/ pRTR-miR-34a, SW480/pRTR-miR-34a, and SW480/pRTR-p53 cells were generated as described previously $(18,26)$. Briefly, cells were transfected with pRTR plasmids using FuGene reagent (Roche). After 24 hours, cells were transferred into medium containing $4 \mu \mathrm{g} / \mathrm{ml}$ puromycin for 1 week. Homogeneity of the derived cell pools was tested by addition of $100 \mathrm{ng} / \mathrm{ml}$ DOX for 48 hours and evaluation of GFP expression by fluorescence microscopy. DLD-1/tTA-p53 cells were described previously (59).

ChIP assay. Crosslinking of cells was performed with $1 \%$ formaldehyde (Merck) and terminated after 5 minutes by addition of glycine at a final concentration of $0.125 \mathrm{M}$. Cells were harvested with SDS buffer $(50 \mathrm{mM}$ Tris pH 8.1, $0.5 \%$ SDS, $100 \mathrm{mM} \mathrm{NaCl}, 5 \mathrm{mM}$ EDTA) and after pelleting resuspended in IP buffer ( 2 parts of SDS buffer and 1 part Triton dilu- 
tion buffer [100 mM Tris- $\mathrm{HCl}$, pH 8.6, $100 \mathrm{mM} \mathrm{NaCl}, 5$ mM EDTA, pH $8.0,0.2 \% \mathrm{NaN}_{3}, 5.0 \%$ Triton X-100]). Chromatin was sheered by 8 sonication cycles (HTU SONI 130; G. Heinemann) to generate DNA fragments with an average size of $700 \mathrm{bp}$ for qChIP. Preclearing and incubation with polyclonal STAT3 antibody (sc-482; Santa Cruz Biotechnology Inc.) or IgG control (M-7023; Sigma-Aldrich) for 16 hours was performed as previously described (60). Washing and reversal of crosslinking was performed as described (61). ChIP-DNA was analyzed by quantitative real-time PCR (qPCR) and the enrichment was expressed as fold enrichment compared with IgG. The sequences of oligonucleotides used as qChIP primers are listed in Supplemental Table 2.

Boyden chamber assays of invasion. To analyze invasion, cell inserts (8.0 $\mu \mathrm{m}$ pore size membrane; Corning) were first coated with Matrigel (BD Bioscience) at a dilution of $3.3 \mathrm{ng} / \mathrm{ml}$ in medium without serum. Subsequently, $5 \times 10^{4}$ cells, previously deprived of serum (0.1\%) for 24 hours, were seeded on the Matrigel in the upper chamber in serum-free medium. Trypsin was inactivated with Trypsin neutralization solution (Clonetics). As a chemoattractant, $20 \%$ FBS was placed in the lower chamber. After 48 hours, nonmotile cells at the top of the filter were removed and the cells in the bottom chamber were fixed with methanol and stained with DAPI and counted using immunofluorescence microscopy. Results represent the average number of cells in 5 fields per membrane in triplicate inserts.

Wound healing scratch assay. Cells were cultured for 24 hours in the presence of DOX (100 ng/ml) and/or IL-6 (20 ng/ml). Mitomycin C (10 ng/ml) was added 2 hours before generating a scratch by removing a Culture-Insert (80241; IBIDI). After washing twice with HBSS to remove Mitomycin C and detached cells, medium containing DOX and/or IL- 6 as indicated was added. Images were captured on an Axiovert Observer Z.1 microscope connected to an AxioCam MRm camera using Axiovision software (Zeiss) at the respective time points.

$R N A$ isolation and $q P C R$. Total RNA was isolated using the Total RNA Isolation Kit (Roche) according to the manufacturer's instructions. For mRNA and primary miRNA analyses, cDNA was generated from $1 \mu \mathrm{g}$ total RNA per sample using the Verso cDNA synthesis kit (Thermo Scientific). qPCR was performed by using the LightCycler 480 (Roche) and the Fast SYBR Green Master Mix (Applied Biosystems). For mature miRNA analyses, cDNA was generated and qPCR was performed using the Exiqon Universal cDNA Synthesis Kit, SYBR Green Master Mix, and commercially available primers (Exiqon). mRNA and miRNA expression were normalized using detection of GAPDH and SNORD48, respectively. Results are represented as fold induction using the $\Delta \Delta \mathrm{Ct}$ method with the control set to 1 as described before (60). The sequences of oligonucleotides used as qPCR primers are listed in Supplemental Table 1.

Western blot analysis and antibodies. Cell lysates were collected in RIPA lysis buffer (50 mM Tris/HCl, pH 8.0, $250 \mathrm{mM} \mathrm{NaCl}, 1 \% \mathrm{NP} 40,0.5 \%$ [w/v] sodium deoxycholate, $0.1 \%$ sodium dodecylsulfate, complete mini protease and phosphatase inhibitors; latter 2 were from Roche). Lysates were sonicated and centrifuged at $16,060 \mathrm{~g}$ for 15 minutes at $4^{\circ} \mathrm{C}$. Per lane, 30-60 $\mu \mathrm{g}$ of whole-cell lysate was separated on $6 \%$ or $12 \%$ SDS-acrylamide gels and transferred on Immobilon PVDF membranes (Millipore). For immunodetection, membranes were incubated with antibodies listed in Supplemental Table 5. Signals from HRP-coupled secondary antibodies were generated by enhanced chemiluminescence (Millipore) and recorded with a CCD camera (440CF imaging system; Eastman Kodak Co.). Intensities of protein expression signals were quantified using densitometric analysis with Kodak Molecular Imaging software v5.0.1.27. The resulting values of the protein of interest were normalized to the corresponding loading controls.

Metastases formation in a xenograft mouse model. DLD-1 and SW620 cells stably expressing Luc2 were generated as described previously (54). Luciferasetagged cells $\left(4 \times 10^{6} / 0.2 \mathrm{ml}\right)$ were injected into the lateral tail vein of NOD/
SCID mice using 25-gauge needles. In weekly intervals, anesthetized mice were injected i.p. with D-luciferin $(150 \mathrm{mg} / \mathrm{kg})$ and imaged 10 minutes after injection using the IVIS Illumina System (Caliper Life Sciences). The acquisition time was 2 minutes. Ten weeks after tail vein injection, mice were sacrificed and examined for lung metastases using H\&E staining.

Immunofluorescence and confocal laser-scanning microscopy. Cells cultivated on glass cover slides were fixed in $4 \%$ paraformaldehyde/PBS for 10 minutes, permeabilized in $0.2 \%$ Triton X-100 for 20 minutes, and blocked in $100 \%$ FBS for 1 hour. Proteins were detected with antibodies listed in Supplemental Table 5. Chromatin was stained by DAPI (Roth). Specimens were covered with ProLong Gold Antifade (Invitrogen). As a negative control, all stainings were performed without primary antibody. Confocal laser scanning microscopy (CLSM) images were captured with a LSM700 microscope using a Plan Apochromat $20 \times / 0.8 \mathrm{M} 27$ objective and ZEN 2009 software (Zeiss) with the following settings: image size, $2048 \times 2048$ and 16 bit; pixel/dwell, $25.2 \mu$ s; pixel size, $0.31 \mu \mathrm{m}$; laser power, $2 \%$; and master gain, $600-1000$. After image capturing, the original CLSM files were converted into TIFF files.

Dual 3' UTR reporter assays. 3' UTRs of human and mouse IL6R were PCR amplified from oligo-dT-primed cDNA of human diploid fibroblasts with the Verso cDNA kit (Thermo Scientific), inserted into pGL3-control-MCS (62), and verified by sequencing. Mutations in the miR-34a seed-matching sequences were generated with the QuikChange Mutagenesis Kit according to the manufacturer's instructions (Stratagene). Oligonucleotides used for cloning and mutagenesis are given in Supplemental Table 3. For luciferase assays, H1299 cells were seeded in 12-well format dishes with $3 \times 10^{4}$ cells/well and transfected after 24 hours with $100 \mathrm{ng}$ of the indicated firefly luciferase reporter plasmid, 20 ng of Renilla reporter plasmid as a normalization control, and $25 \mathrm{nM}$ of miR-34a or miR-34c pre-miRNA (PM11030, PM11039; Ambion) or a negative control oligonucleotide (neg. control \#1; Ambion). After 48 hours, a Dual Luciferase Reporter assay (Promega) was performed according to the manufacturer's instructions. Fluorescence intensities were measured with an Orion II luminometer (Berthold) in 96-well format and analyzed with the SIMPLICITY software package (DLR).

Analysis of human CRC samples. The human colon cancer collection consisted of surgically resected invasive CRC specimens from 48 patients; specimens were obtained from the biobank of the Klinikum Rechts der Isar and the medical faculty of the Technical University Munich (BBTU). RNA isolation was done on freshly frozen material as described previously (10). Immunohistochemistry was done using 5 - $\mu \mathrm{m}$ sections of formalinfixed, paraffin-embedded tissue of the same tumor specimens. AntiY705-p-STAT3 (Cell Signaling Technologies, dilution 1:50) was used as the primary antibody, and staining was done on a Ventana Benchmark XT Autostainer applying the XT UltraView Diaminobenzidine Kit (Ventana Medical Systems) and hematoxylin for counterstaining. p-Stat3 expression was semiquantitatively scored in a blinded fashion as low or high based on nuclear staining intensity of tumor cells.

TCGA expression data from colon adenocarcinomas of 425 patients was obtained from the TCGA data portal (ref. 32; https://tcga-data.nci.nih. gov/tcga/tcgaDownload.jsp). The expression of mRNAs had been determined by next generation sequencing data from RNAseq HiSeqV2 3.1.2.0 and GASeqV2 3.1.0.0 databases, whereas expression of miRNAs was from miRNASeq HiSeq 3.1.2.0 and GASeq 3.1.2.0 sample sets. Expression levels are displayed as normalized number of counts.

Generation and handling of mice. Mir34a-/- mice (SV/C57BL6 background) were generated using homologous recombination with a vector containing the Mir34a seed flanked with loxP sites and an intronic neomycin resistance cassette flanked by frt sites (Figure 7, E and F, and Supplemental Figure 7). The Neo cassette was removed by crossing with flp-mice and Mir34a by crossing with CMV-Cre mice. As a control, mice homozygous for Mir34a flanked with loxP sites were used. Oligonucleotides for genotyping 


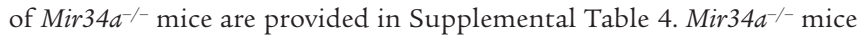
showed no obvious phenotype and were born at a normal Mendelian ratio. Six- to ten-week-old Mir34a-/- and Mir $34 a^{F / F}$ mice were used for experiments. Six- to eight-week-old age-matched male immunocompromised NOD/SCID mice were purchased from Jackson Laboratories and used for lung metastasis assays after tail-vein injection.

CAC induction and analysis in mice. AOM/DSS-induced tumorigenesis was performed essentially as described with slight modifications (63). Injections (i.p.) of $10 \mathrm{mg} / \mathrm{kg} \mathrm{AOM} \mathrm{(Sigma-Aldrich)} \mathrm{were} \mathrm{per-}$ formed once at day 0 and once at day 21 (Figure 7D). Five days after AOM injection, mice received 2\% DSS (MP Biosystems) in the drinking water for 5 days, followed by 16 days of regular water (first and second cycles). During the third cycle, no AOM and only $2 \%$ DSS were administered. At day 110, mice were sacrificed. The colon was opened longitudinally and rolled to form a Swiss roll (64). Tissues were fixed overnight in $4 \%$ paraformaldehyde/PBS (Santa Cruz Biotechnology Inc.), dehydrated, and embedded in paraffin; $3-\mu \mathrm{m}$ sections were obtained. Tumor counts were performed in a blinded fashion. Tumor sizes were calculated by using AxioVision Rel. 4.8 software (Zeiss). For proliferation rates, mice were i.p. injected with BrdU (Amersham) at $75 \mathrm{mg} / \mathrm{kg}$ in PBS 1.5 hours before they were sacrificed. For Western blot analysis, tumor tissue was lysed in ice-cold lysis buffer by mechanical homogenization as described previously (63). To complete lysis, samples were incubated 10 minutes on ice and centrifuged at $15,000 \mathrm{~g}$ at $4^{\circ} \mathrm{C}$ to separate cell debris and the lysate. Immunohistological analyses were performed as described elsewhere (39) using antibodies listed in Supplemental Table 5. Tumor cells with positive IHC staining for cleaved caspase 3, p-STAT3, and SNAIL as well as BrdU were enumerated in high-power fields (HPF) from similar size tumors were counted. The expression of IL- 6 was evaluated as follows: percentage of positive tumor cells was multiplied by the staining intensity (score: 1 , weak staining; 2 , moderate staining; or 3 , strong staining). To analyze mutations in the TP53 gene, cDNAs generated from RNA isolated from tumor lysates were sequenced as described elsewhere (65). All coding exons of 5 to 10 tumors from each Mir34a genotype were sequenced.
Statistics. Data were expressed as mean \pm SD or \pm SEM. Differences were analyzed by a 2-tailed Student's $t$ test or 1-way ANOVA with Tukey multiple comparison post test in cases in which more than 2 conditions were compared on the same graph. Calculations were performed using Prism 5 (Graph Pad Software Inc.) and $P$ values of 0.05 or less were considered as significant.

Study approval. All animal protocols were approved by the Regierung von Oberbayern. All procedures involving human tumor biopsies were performed with the approval of the ethics committee of the Technical University of Munich on samples from patients who had given written informed consent.

\section{Acknowledgments}

We thank Stefanie Jaitner for NOD/SCID mice, Andrea Sendelhofert and Anja Heier for support with IHC staining, and Sabine Hünten and Helge Siemens for CRC cell pools with inducible $m i R-34 a$ and $p 53$ expression (Institute of Pathology, Ludwig-Maximilians-University Munich) and Bert Vogelstein (Johns Hopkins Medical School, Baltimore, Maryland, USA) for HCT116 TP53-/and DLD-1/tTa-p53 cells. This work was supported by grants from the Deutsche Krebshilfe (109531), the DKTK, and the RudolfBartling-Foundation (to Heiko Hermeking), and by grants from the Deutsche Forschungsgemeinschaft (Gr1916/3-1) and the European Research Council (ROSCAN-281967) to Florian R. Greten.

Received for publication October 2, 2013, and accepted in revised form January 9, 2014.

Address correspondence to: Heiko Hermeking, Experimental and Molecular Pathology, Institute of Pathology, Ludwig-MaximiliansUniversität München, Thalkirchner Strasse 36, D-80337 Munich, Germany. Phone: 49.89.2180.73685; Fax: 49.89.2180.73697; E-mail: heiko.hermeking@med.uni-muenchen.de. Or to: Florian R. Greten, Georg-Speyer-Haus, Institute for Tumor Biology and Experimental Therapy, Paul-Ehrlich-Str. 42-44, 60596 Frankfurt am Main, Germany. Phone: 49.69.63395.232; Fax: 49.69.63395.184; E-mail: greten@gsh.uni-frankfurt.de.
1. Gupta GP, Massague J. Cancer metastasis: building a framework. Cell. 2006;127(4):679-695.

2. Brabletz T, et al. Invasion and metastasis in colorectal cancer: epithelial-mesenchymal transition, mesenchymal-epithelial transition, stem cells and betacatenin. Cells Tissues Organs. 2005;179(1-2):56-65.

3. Thiery JP, Acloque H, Huang RY, Nieto MA. Epithelial-mesenchymal transitions in development and disease. Cell. 2009;139(5):871-890.

4. Kalluri R, Weinberg RA. The basics of epithelial-mesenchymal transition. J Clin Invest. 2009; 119(6): 1420-1428

5. Lopez-Novoa JM, Nieto MA. Inflammation and EMT: an alliance towards organ fibrosis and cancer progression. EMBO Mol Med. 2009;1(6-7):303-314.

6. Balkwill F, Charles KA, Mantovani A. Smoldering and polarized inflammation in the initiation and promotion of malignant disease. Cancer Cell. 2005;7(3):211-217.

7. Hussain SP, Harris CC. Inflammation and cancer: an ancient link with novel potentials. Int J Cancer. 2007;121(11):2373-2380.

8. Terzic J, Grivennikov S, Karin E, Karin M. Inflammation and colon cancer. Gastroenterology. 2010; 138(6):2101-2114

9. Quante M, Varga J, Wang TC, Greten FR. The gastrointestinal tumor microenvironment. Gastroenterology. 2013;145(1):63-78.

10. Schwitalla $S$, et al. Loss of p53 in enterocytes generates an inflammatory microenvironment enabling invasion and lymph node metastasis of carcinogen-induced colorectal tumors. Cancer Cell. 2013;23(1):93-106.

11. Grivennikov SI, et al. Adenoma-linked barrier defects and microbial products drive IL-23/ IL-17-mediated tumour growth. Nature. 2012; 491(7423):254-258.

12. Grivennikov SI, Karin M. Inflammatory cytokines in cancer: tumour necrosis factor and interleukin 6 take the stage. Ann Rheum Dis. 2011;1:1104-i108.

13. Knupfer H, Preiss R. Serum interleukin-6 levels in colorectal cancer patients--a summary of published results. Int J Colorectal Dis. 2010;25(2):135-140.

14. Lujambio A, Lowe SW. The microcosmos of cancer. Nature. 2012;482(7385):347-355.

15. Brabletz T. MiR-34 and SNAIL: another double-negative feedback loop controlling cellular plasticity/EMT governed by p53. Cell Cycle. 2012;11(2):215-216.

16. Hermeking H. MicroRNAs in the p53 network: micromanagement of tumour suppression. Nat Rev Cancer. 2012;12(9):613-626.

17. Hermeking H. p53 enters the microRNA world. Cancer Cell. 2007;12(5):414-418.

18. Siemens H, et al. miR-34 and SNAIL form a doublenegative feedback loop to regulate epithelial-mesenchymal transitions. Cell Cycle. 2011;10(24):4256-4271.

19. $\mathrm{Kim} \mathrm{NH}$, et al. A p53/miRNA-34 axis regulates Snail1-dependent cancer cell epithelial-mesenchymal transition. J Cell Biol. 2011;195(3):417-433.

20. Iorio MV, Croce CM. microRNA involvement in human cancer. Carcinogenesis. 2012;33(6):1126-1133.
21. Ebert MS, Sharp PA. Roles for microRNAs in conferring robustness to biological processes. Cell. 2012;149(3):515-524.

22. Ptashne M. Binding reactions: epigenetic switches, signal transduction and cancer. Curr Biol. 2009;19(6):R234-R241.

23. Hitchler MJ, Domann FE. Metabolic defects provide a spark for the epigenetic switch in cancer. Free Radic Biol Med. 2009;47(2):115-127.

24. Rokavec M, Wu W, Luo JL. IL6-Mediated Suppression of miR-200c Directs Constitutive Activation of Inflammatory Signaling Circuit Driving Transformation and Tumorigenesis. Mol Cell. 2012;45(6):777-789.

25. Iliopoulos D, Hirsch HA, Struhl K. An epigenetic switch involving NF-kappaB, Lin28, Let-7 MicroRNA, and IL6 links inflammation to cell transformation. Cell. 2009;139(4):693-706.

26. Jackstadt R, et al. AP4 is a mediator of epithelialmesenchymal transition and metastasis in colorectal cancer. J Exp Med. 2013;210(7):1331-1350.

27. Qin H, et al. Signal transducer and activator of transcription-3/suppressor of cytokine signaling-3 (STAT3/SOCS3) axis in myeloid cells regulates neuroinflammation. Proc Natl Acad Sci U S A. 2012;109(13):5004-5009.

28. Moreno-Bueno G, et al. The morphological and molecular features of the epithelial-to-mesenchymal transition. Nat Protoc. 2009;4(11):1591-1613.

29. De Craene B, Berx G. Regulatory networks defining EMT during cancer initiation and progression. Nat 
Rev Cancer. 2013;13(2):97-110.

30. Becker C, et al. TGF-beta suppresses tumor progression in colon cancer by inhibition of IL- 6 transsignaling. Immunity. 2004;21(4):491-501.

31. Mukhopadhyay UK, Mooney P, Jia L, Eves R, Raptis L, Mak AS. Doubles game: Src-Stat3 versus p53PTEN in cellular migration and invasion. Mol Cell Biol. 2010;30(21):4980-4995.

32. Cancer Genome Atlas Network. Comprehensive molecular characterization of human colon rectal cancer. Nature. 2012;487(7407):330-337.

33. Neufert C, Becker C, Neurath MF. An inducible mouse model of colon carcinogenesis for the analysis of sporadic and inflammation-driven tumor progression. Nat Protoc. 2007;2(8):1998-2004.

34. Takahashi M, Nakatsugi S, Sugimura T, Wakabayashi K. Frequent mutations of the beta-catenin gene in mouse colon tumors induced by azoxymethane. Carcinogenesis. 2000;21(6):1117-1120.

35. Tanaka T, Kohno H, Suzuki R, Yamada Y, Sugie $\mathrm{S}$, Mori $\mathrm{H}$. A novel inflammation-related mouse colon carcinogenesis model induced by azoxymethane and dextran sodium sulfate. Cancer Sci. 2003;94(11):965-973

36. Grivennikov SI, Greten FR, Karin M. Immunity, inflammation, and cancer. Cell. 2010;140(6):883-899.

37. Fearon ER, Vogelstein B. A genetic model for colorectal tumorigenesis. Cell. 1990;61(5):759-767.

38. Schwitalla $S$, et al. Intestinal Tumorigenesis Initiated by Dedifferentiation and Acquisition of StemCell-like Properties. Cell. 2013;152(1-2):25-38.

39. Bollrath J, et al. gp130-mediated Stat 3 activation in enterocytes regulates cell survival and cell-cycle progression during colitis-associated tumorigenesis. Cancer Cell. 2009;15(2):91-102.

40. Grivennikov S, et al. IL-6 and Stat 3 are required for survival of intestinal epithelial cells and development of colitis-associated cancer. Cancer Cell. 2009;15(2):103-113.

41. Yu H, Pardoll D, Jove R. STATs in cancer inflammation and immunity: a leading role for STAT3. Nat Rev Cancer. 2009;9(11):798-809.

42. Tsai JH, Donaher JL, Murphy DA, Chau S, Yang J. Spatiotemporal regulation of epithelial-mesenchymal transition is essential for squamous cell carcinoma metastasis. Cancer Cell. 2012;22(6):725-736.

43. Spehlmann ME, et al. Trp53 deficiency protects against acute intestinal inflammation. J Immunol. 2013;191(2):837-847.

44. Christoffersen NR, et al. p53-independent upregulation of miR-34a during oncogene-induced senescence represses MYC. Cell Death Differ. 2010; 17(2):236-245.

45. Feng Y, et al. Heat shock improves Sca-1(+) stem cells survival and directs ischemic cardiomyocytes toward a prosurvival phenotype via exosomal transfer: a critical role for HSF1/miR-34a/HSP70 pathway. Stem Cells. 2014;32(2):462-472.

46. Xiong H, et al. Roles of STAT3 and ZEB1 proteins in E-cadherin down-regulation and human colorectal cancer epithelial-mesenchymal transition. J Biol Chem. 2012;287(8):5819-5832.

47. Lyons JG, et al. Snail up-regulates proinflammatory mediators and inhibits differentiation in oral keratinocytes. Cancer Res. 2008;68(12):4525-4530.

48. Yadav A, Kumar B, Datta J, Teknos TN, Kumar P. IL-6 promotes head and neck tumor metastasis by inducing epithelial-mesenchymal transition via the JAK-STAT3-SNAIL signaling pathway. Mol Cancer Res. 2011;9(12):1658-1667.

49. Studebaker AW, et al. Fibroblasts isolated from common sites of breast cancer metastasis enhance cancer cell growth rates and invasiveness in an interleukin-6-dependent manner. Cancer Res. 2008;68(21):9087-9095.

50. Li Y, et al. MicroRNA-34a inhibits glioblastoma growth by targeting multiple oncogenes. Cancer Res. 2009;69(19):7569-7576.

51. Siemens H, Jackstadt R, Kaller M, Hermeking H. Repression of c-Kit by p53 is mediated by miR34 and is associated with reduced chemoresistance, migration, and stemness. Oncotarget. 2013; 4(9):1399-1415.

52. Mudduluru G, Ceppi P, Kumarswamy R, Scagliotti GV, Papotti M, Allgayer H. Regulation of Axl receptor tyrosine kinase expression by miR$34 \mathrm{a}$ and $\mathrm{miR}-199 \mathrm{a} / \mathrm{b}$ in solid cancer. Oncogene. 2011;30(25):2888-2899.

53. Li N, et al. miR-34a inhibits migration and invasion by down-regulation of c-Met expression in human hepatocellular carcinoma cells. Cancer Lett. 2009;275(1):44-53.

54. Hahn S, Jackstadt R, Siemens H, Hünten S, Hermeking H. SNAIL miR-34a feed-forward regulation of
ZNF281/ZBP99 promotes epithelial-mesenchymal transition. EMBO J. 2013;32(23):3079-3095.

55 . Rose-John S. IL-6 trans-signaling via the soluble IL-6 receptor: importance for the pro-inflammatory activities of IL-6. Int J Biol Sci. 2012;8(9):1237-1247.

56. Matsumoto S, et al. Essential roles of IL- 6 transsignaling in colonic epithelial cells, induced by the IL-6/soluble-IL-6 receptor derived from lamina propria macrophages, on the development of colitis-associated premalignant cancer in a murine model. J Immunol. 2010;184(3):1543-1551.

57. Siemens H, et al. Detection of miR-34a Promoter Methylation in Combination with Elevated Expression of c-Met and beta-Catenin Predicts Distant Metastasis of Colon Cancer. Clin Cancer Res. 2013; 19(3):710-720.

58. Rose-John S, Waetzig GH, Scheller J, Grotzinger J, Seegert D. The IL-6/sIL-6R complex as a novel target for therapeutic approaches. Expert Opin Ther Targets. 2007;11(5):613-624.

59. Yu J, Zhang L, Hwang PM, Rago C, Kinzler KW, Vogelstein B. Identification and classification of p53-regulated genes. Proc Natl Acad Sci US A. 1999; 96(25):14517-14522.

60. Menssen A, et al. c-MYC delays prometaphase by direct transactivation of MAD2 and BubR1: identification of mechanisms underlying c-MYCinduced DNA damage and chromosomal instability. Cell Cycle. 2007;6(3):339-352.

61. Frank SR, Schroeder M, Fernandez P, Taubert S, Amati B. Binding of c-Myc to chromatin mediates mitogen-induced acetylation of histone $\mathrm{H} 4$ and gene activation. Genes Dev. 2001;15(16):2069-2082.

62. Welch C, Chen Y, Stallings RL. MicroRNA-34a functions as a potential tumor suppressor by inducing apoptosis in neuroblastoma cells. Oncogene. 2007;26(34):5017-5022.

63. Greten FR, et al. IKKbeta links inflammation and tumorigenesis in a mouse model of colitis-associated cancer. Cell. 2004;118(3):285-296.

64. Moolenbeek C, Ruitenberg EJ. The "Swiss roll": a simple technique for histological studies of the rodent intestine. Lab Anim. 1981;15(1):57-59.

65. Jerry DJ, Ozbun MA, Kittrell FS, Lane DP, Medina D, Butel JS. Mutations in p53 are frequent in the preneoplastic stage of mouse mammary tumor development. Cancer Res. 1993;53(14):3374-3381. 